\title{
Hydrodynamics of a rigid stationary flat plate in cross-flow near the free surface
}

Cite as: Phys. Fluids 31, 102108 (2019); https://doi.org/10.1063/1.5111525

Submitted: 29 May 2019 . Accepted: 02 October 2019 . Published Online: 30 October 2019

H. R. Díaz-Ojeda (D) F. J. Huera-Huarte (D), and L. M. González-Gutiérrez (D)

\section{COLLECTIONS}

EP This paper was selected as an Editor's Pick
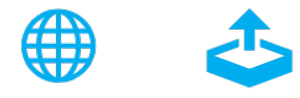

\section{ARTICLES YOU MAY BE INTERESTED IN}

Deformation of a vortex ring caused by its impingement on a sphere Physics of Fluids 31, 107108 (2019); https://doi.org/10.1063/1.5122260

Modification of three-dimensional instability in the planar shear flow around two circular cylinders in tandem

Physics of Fluids 31, 104110 (2019); https://doi.org/10.1063/1.5120564

Effective viscoelasticity of non-Newtonian fluids modulated by large-spherical particles aligned under unsteady shear

Physics of Fluids 31, 103304 (2019); https://doi.org/10.1063/1.5119335

\section{AIP Author Services English Language Editing}

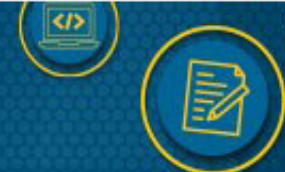




\title{
Hydrodynamics of a rigid stationary flat plate in cross-flow near the free surface ${ }^{\circledR}$
}

\author{
Cite as: Phys. Fluids 31, 102108 (2019); doi: 10.1063/1.5111525 \\ Submitted: 29 May 2019 - Accepted: 2 October 2019 • \\ Published Online: 30 October 2019
}

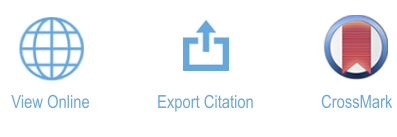

\author{
H. R. Díaz-Ojeda,' (D) F. J. Huera-Huarte, ${ }^{2}$ (D) and L. M. González-Gutiérrez ${ }^{1, a)}$ (D)
}

\author{
AFFILIATIONS \\ ${ }^{7}$ CEHINAV, ETSIN, Universidad Politécnica de Madrid (UPM), Avenida de la Memoria 4, 28040 Madrid, Spain \\ ${ }^{2}$ Department of Mechanical Engineering, Universitat Rovira i Virgili, 43007 Tarragona, Spain
}

a) Author to whom correspondence should be addressed: leo.gonzalez@upm.es

\begin{abstract}
In this work, the flow normal to a rectangular flat plate with sharp angles in the presence of the free surface and gravity has been studied numerically, using a three dimensional (3D) large Eddy simulation methodology. Most of the previous studies on this geometry consider either periodic assumptions or laminar regime $R e \sim O\left(10^{3}\right)$. The numerical study described here, with a fully $3 \mathrm{D}$ simulation of the flat plate in turbulent regime $R e \sim O\left(10^{5}\right)$, had not been reported earlier. Important differences have been found with respect to the laminar case or when periodic boundary conditions are assumed. The influence of the gravity force and the free surface interface on the hydrodynamics of the problem is extremely relevant for industrial applications. The simulations are initially validated using a reference case with a plate with sharp corners and a single phase laminar 3D-periodic configuration. The time averaged drag force, the velocity, and pressure fields are compared as well against the case of the plate with smooth corners. Finally, in the case of the two-phase 3D numerical simulations, the drag force and the vorticity fields in the near wake of the plate are compared to recent experimental work [S. Satheesh and F. J. Huera-Huarte, "Effect of the free surface on a flat plate translating normal to the flow," Ocean Eng. 171, 458-468 (2019)]. These experiments were used as a guideline for the computational setup; consequently, the values of most of the dimensionless parameters are the same. A second scenario where the free surface is replaced by a solid wall is considered. Two important observations show up after this research: first, the characteristic unsteady frequencies that are found associated with two periodic regimes disappear when $3 D$ cases are evaluated. In such a scenario, no dominant frequencies are found. Second, a critical submergence depth (distance) between the upper part of the plate and the free surface (or solid wall) has been found, where the drag force shows a maximum value. These observations are discussed in relation to the pressure distribution, the vortex structures formed at the wake, and the gap flow formed between the plate and the free surface.
\end{abstract}

Published under license by AIP Publishing. https://doi.org/10.1063/1.5111525

\section{INTRODUCTION}

Canonical bluff bodies such as circular or rectangular cylinders have important implications and significant relevance in engineering problems. They are frequently found, for instance, in energyharvesting devices that extract energy from the ocean waves and sea currents, ocean structures, bridges, offshore rigs, etc. Most of these complex engineering applications involve the presence of the free surface as well. In the past, researchers have dealt with such problems numerically, using different simplifications, i.e., limiting geometries to 2D domains, using single phase models, or assuming low Reynolds numbers that imply the absence of turbulence. Sheridan et al. (1997) and Reichl et al. (2005) studied the influence of the free surface in a 2D submerged circular cylinder. They both showed the strong asymmetry created by the free surface in the vortex dynamics. These authors observed that if the free surface is taken into account, the Froude number defined as $F r=\frac{U}{\sqrt{g D}}$, where $U$ is the free stream velocity flow, $g$ is the gravity acceleration, and $D$ is the cylinder diameter, is crucial in order to understand the problem. Other numerical methods such as Smooth Particle Hydrodynamics ( $\mathrm{SPH}$ ) were used by Bouscasse et al. (2017) who continued the work started by Reichl et al. (2005), studying the wake behavior at a Reynolds number of 180. In those works, different free surface deformations were observed depending on the Froude number, where supercritical to subcritical 
transitions take place in the near wake resulting in localized freesurface sharpening and wave breaking. Miyata et al. (1990) studied the fluid forces and the free surface influence on a submerged circular cylinder in turbulent regime $R e=4.96 \times 10^{4}$. Numerical simulations and experiments were performed showing a simultaneous decrease in the drag coefficient and an increase in the Strouhal number when the depth of the circular cylinder was gradually reduced. Malavasi and Guadagnini (2007) showed experimentally the influence on the hydrodynamic forces of the relative position of a rectangular cylinder, asymmetrically placed between the free surface and the channel floor. This effect had a large impact on the transverse force but barely affected the drag coefficient. The influence of the free surface on the fluid-structure interactions showed by a circular cylinder with an elastic plate has been recently studied, at Reynolds numbers in the range from 100 to 1000 , by Díaz-Ojeda et al. (2018; 2019).

Different numerical approaches for solving the wake behind a normal flat plate have been used in the past. Najjar and Balachandar (1998) studied numerically the 3D-periodic version of a flat plate in a laminar regime $(R e=250)$ in a single phase flow and captured low-frequencies unsteadiness with periods approximately 10 times smaller than the primary shedding period. This behavior was associated with two different flow states: an $\mathrm{H}$ regime of high mean drag and an L regime of low mean drag. Narasimhamurthy and Andersson (2009) solved the same problem in the laminar regime using Direct Numerical Simulations (DNSs) at a Reynolds number of 750, exploring the coupling between the base pressure and the vortex formation process. Hemmati et al. (2016b) compared simulations of 3D-periodic flow against 3D flow, for a particular aspect ratio flat plate with sharp corners and $R e=1200$. Note that $3 \mathrm{D}$-periodic means that the computational domain is $3 \mathrm{D}$, but there is a periodic boundary condition on the spanwise direction which implies the constant $\mathrm{z}$ planes; also, the length of the computational domain in the $\mathrm{Z}$ direction is equal to the spanwise length of the plate. The results they obtained showed very different features in terms of wake dynamics, vortex structures, frequencies in the wake, and averaged drag values. Apparently, the presence of sideedge shear layers suppressed the spanwise instabilities responsible for the 3 distinct flow regimes that were observed in the wake of $3 \mathrm{D}$-periodic plates. A vortex peeling mechanism linked to the vortex detachments in the shear layers on the shorter sides seems to appear only in 3D flows. Moreover, the peeling mechanism leads to the formation of interlocked vortex loops outside the base region. Another important finding was the single dominant shedding frequency observed in the wake, which indicated that the two shear layers were rolling up at the same frequency at adjacent plate edges. In the work of Hemmati et al. (2016a), the 3D-periodic normal flat plate was studied again using DNS at Reynolds numbers of 1200 and 2400, and a distinct period of reorganization $\mathrm{M}$ was identified following regime $\mathrm{H}$.

In the work of Hemmati et al. (2018), the same 3D-periodic problem is studied using DNS and large Eddy simulation (LES) for a extended Reynolds range up to $R e=1.5 \times 10^{5}$, where the author suggests that for $R e>1000$, the influence of the Reynolds number on the drag and pressure coefficient as well as on the Strouhal number and the mean recirculation length was limited.

Tian et al. (2014) studied numerically the 3D-periodic case using a LES model at turbulent Reynolds numbers of $1.5 \times 10^{5}$, in a plate with smooth corners. This work showed that the corner configuration and the reduction in the radius of curvature of the plate edges heavily altered the flow patterns. It increased the mean and the fluctuating part of the drag and the lift forces on the plate, as well as the kinetic energy in the near wake. The work complements the previous research performed by Tian et al. (2012) and Najjar and Balachandar (1998) where the authors showed how their 2D simulations resulted in different hydrodynamic forces when compared to 3D LES or experimental results.

Although most of the studies performed with rectangular plates have been carried out without considering the free surface (a single phase flow), some exceptions are found in the literature. One of the numerical studies that includes free surface is the work of Liu et al. (2016). This 2D RANS study, based on the $k-\omega$ SST model at a Reynolds number of $5 \times 10^{4}$, considers the free surface influence on the plate dynamics for turbulent flows with a free surface. Even at their highest submergence depth case, where the free surface influence is expected to be minimal or negligible, the authors showed different mean drag values when compared to those found in other numerical studies, in which the free surface was not taken into account (Tian et al., 2014). Despite the geometry is different, it shows similar vortex dynamics and similar drag trends with depth, as shown in the work of Miyata et al. (1990). When the cylinder is placed closer to the free surface, the drag force decreases and the dimensionless frequency increases.

Satheesh and Huera-Huarte (2019) found a relevant dependence on the aspect ratio and submergence depth, when they studied experimentally rigid plates of different aspect ratios, at Reynolds numbers of $3 \times 10^{4}, 4.5 \times 10^{4}$, and $6 \times 10^{4}$, near the free surface. In general, they have showed how the drag force acting on the plate has a maximum value when near the free surface as the gap flow modifies the wake reducing recirculation length. They also showed that the mean drag force was independent of the Reynolds number, except when the aspect ratios of the plate (span to chord ratio) were in the range from 0.625 to 1.6 and the plate was near the free surface.

In this work, we study the problem of the hydrodynamics of a flat plate normal to the flow in the turbulent regime, considering the effect of the free surface. This is, in fact, a poorly understood canonical problem with great industrial implications that has not received the deserved attention, neither experimentally nor numerically. Even without free surface, very few results have been found for practical Reynolds numbers $R e>10^{4}$ and $3 \mathrm{D}$ isolated plates. Furthermore, questions still remain related to the importance of the free surface in the problem and how much it could affect this kind of flow, which is crucial for marine applications such as energy-harvesting devices. Very few works have been found where simple geometries are tested in the turbulent regime with the free surface, compared to the cases with a single fluid.

The paper is organized as follows: First, the problem description is presented in 2 where the mesh details and the main parameters are introduced, together with the computational methodology (described in Sec. III). In Sec. IV, the methodology is validated for the 3D-periodic case as in the work of Tian et al. (2014). The effects of the free surface on the drag and lift forces, on the pressure field, and on different hydrodynamic aspects are discussed. Finally, concluding remarks are presented in Sec. VI. 


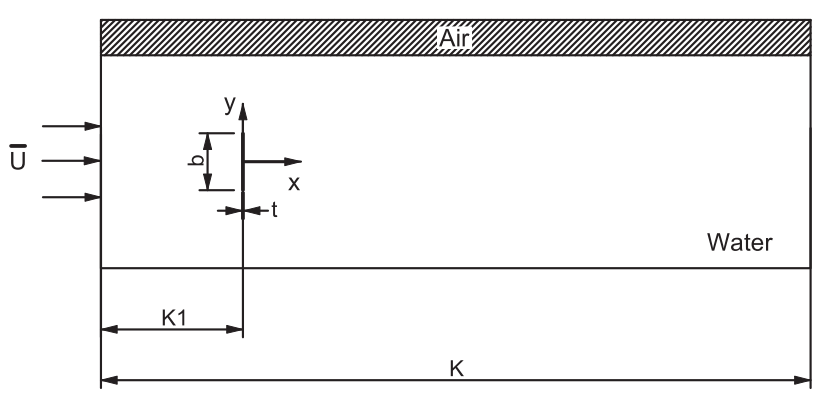

\section{PROBLEM DESCRIPTION}

The dimensions of the computational domain, depicted in Fig. 1 , are $K, G$, and $J$ in the streamwise $(x)$, chordwise $(y)$, and spanwise $(z)$ directions, respectively. The cross section of the plate has a height $b$ ( $y$-axis) and a width $c$ ( $z$-axis) with a thickness $t \ll b, c$. The center of coordinates is located at the center of the plate. We have studied two different cases, a 3D-periodic case with a single phase and a pure $3 \mathrm{D}$ normal flat plate. The $3 \mathrm{D}$ case is studied using two different configurations with a single phase flow (no free-surface but a rigid wall) and a two phase flow (free surface). The results obtained with the last configurations are compared with recent experiments (Satheesh and Huera-Huarte, 2019). The height of the air phase, if present, is $R / b>1$. When two immiscible Newtonian fluids, air and water, are involved in the problem, gravity and free surface are obviously a part of the problem.

Different meshes were studied in order to have an accurate computation of the different flow fields produced and converged measurements of the forces acting on the plate. The mesh structure is always the same as depicted in Fig. 2, with the mesh size and consequently the number of cells varied to obtain the different meshes used. The number of cells on each side depends on the value a single mesh parameter which increases the number of cells. The mesh strategy used for the $3 \mathrm{D}$-periodic case is the same as in the $3 \mathrm{D}$ case with

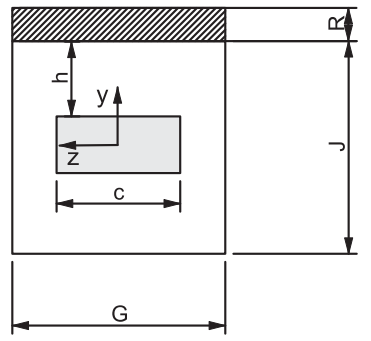

FIG. 1. Schematic of the computational domain. The air phase is only present in the case that involves free surface simulations, not in the case with the rigid wall. the addition of the air phase in the upper domain. Notwithstanding the mesh convergence study, a time step study was also performed. We conclude that a time step $\Delta t>1 \times 10^{-3}$ induces numerical instabilities that lead failures in the simulation. The time step selected for all the simulations is $\Delta t=5 \times 10^{-3}$, leading to errors that are similar to those obtained with smaller time steps, but less expensive from a computational point of view.

We use \langle\rangle$_{t}$ to indicate time averaged quantities, \langle\rangle$_{s}$ for spanwise averaged values, and \langle\rangle when quantities are spatiotemporally averaged. The \langle\rangle average is performed in two steps. First, the time average is performed for each cell and then a spatial average on the selected cells with the previously time averaged values. Both averages are computed as arithmetic mean values.

\section{METHODOLOGY}

The problem described in Sec. II is studied numerically using the free source code OpenFOAM (2018). This code uses a spatial discretization based on the Finite Volume Method (FVM).

First, the case of a flat plate in cross-flow is used as a validation exercise, in order to test the computational tool. After this initial validation, the geometry proposed in the experiments performed by Satheesh and Huera-Huarte (2019) was chosen for a comparative
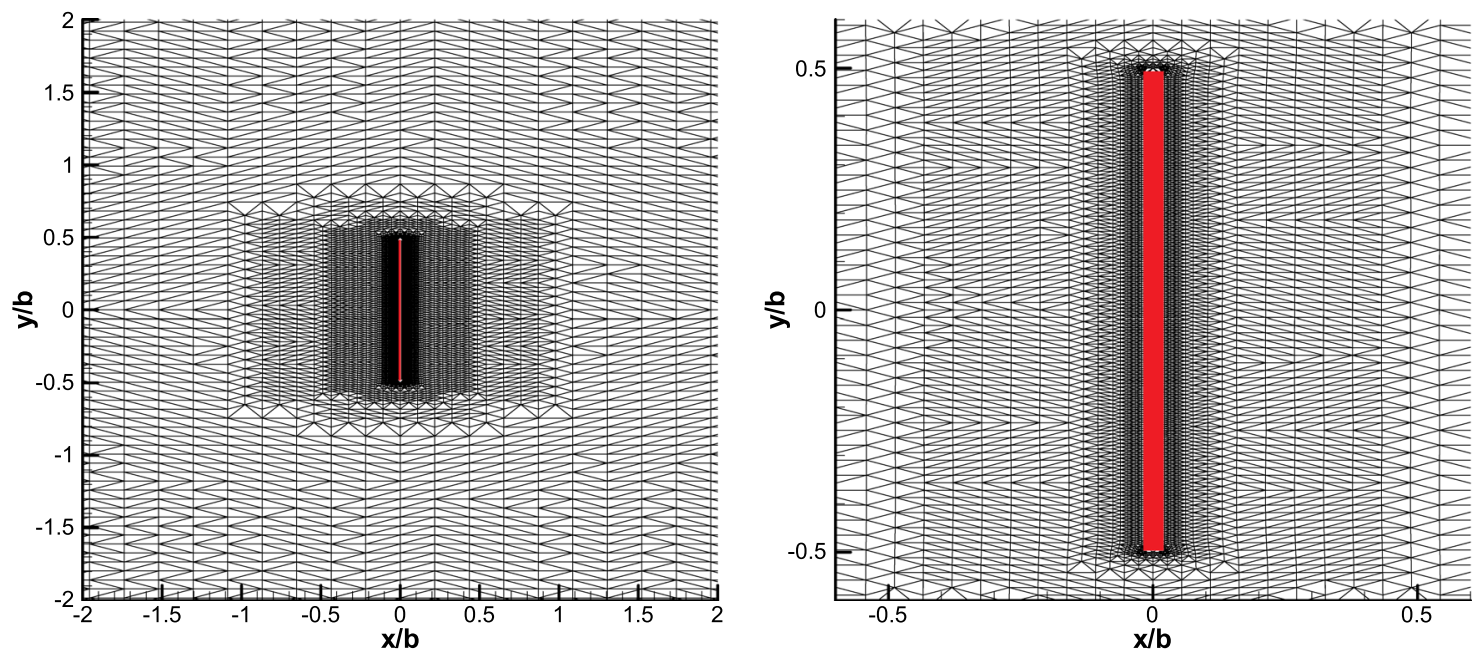

FIG. 2. Scheme of the computational domain for the plane $z=0$. 
TABLE I. Summary of the different parameters of the geometries proposed by Tian et al. (2014) and Satheesh and Huera-Huarte (2019) and the ones used in this work; see Fig. 1. For the case proposed in the work of Satheesh and Huera-Huarte (2019), G/b and J/b are nondimensionalized with the height of the plate $b=0.16 \mathrm{~m}$

\begin{tabular}{|c|c|c|c|c|c|c|c|c|c|}
\hline & $b(\mathrm{~m})$ & $t / b$ & $K_{1} / b$ & $K / b$ & $c / b$ & $d / b$ & $G / b$ & $J / b$ & $\mathrm{AR}$ \\
\hline Tian et al. (2014) & 1 & 0.02 & 7.5 & 27.5 & 4 & & 4 & 16 & 0.25 \\
\hline Satheesh and Huera-Huarte (2019) & $0.16,0.115$ & 0.0063 & 2.5 & 12.5 & 1,2 & $0-1.25$ & 3.75 & 3.75 & $0.5,1$ \\
\hline
\end{tabular}

study; see Fig. 1. A summary of the different geometrical parameters used appears in Table I.

\section{A. Fluid dynamics solver}

The equations to be solved for the fluid phases are the incompressible Navier-Stokes equations. Large-Eddy simulation (LES) is used to model the subgrid scale (SGS) in the present study. This technique solves large scale motions that contain most of the turbulent kinetic energy. Small scale eddies are modeled with a subgrid scale (SGS) model to represent the effects of unresolved motions on the resolved scales. LES uses a spatial average of the transport equations using a filter of size $\Delta$ that separates the large scales solved and the small scales modeled.

In order to define a velocity and pressure field that has only the large fluctuations of the instantaneous velocity, the variables of the Navier-Stokes equations are filtered providing a local mean of the turbulent flow.

Therefore, the filtered incompressible LES equations for the filtered variables are

$$
\begin{aligned}
\nabla \cdot \overline{v_{i}} & =0, \\
\frac{\vartheta\left(\rho \overline{v_{i}}\right)}{\vartheta t}+\nabla\left(\rho \overline{v_{i}} \overline{v_{j}}\right) & =-\nabla \bar{p}+\nabla\left(\mu \nabla \overline{v_{i}}\right)-\nabla \tau_{i j},
\end{aligned}
$$

where the term $\tau_{i j}=\rho\left[\overline{v_{i} v_{j}}-\overline{v_{i}} \overline{v_{j}}\right]$ is modeled. The algebraic model chosen is that developed by Smagorinsky and Lilly (Smagorinsky, 1963), which computes directly the SGS stresses $\tau_{i j}$ from the filtered variables with the following formulation:

$$
\tau_{i j}-\frac{1}{3} \tau_{k k} \delta_{i j}=\mu_{s g s} \bar{S}_{i j},
$$

where Einstein convention is used for repeated indices. Furthermore, $\bar{S}_{i j}$ is the mean strain rate tensor $\bar{S}_{i j}=\frac{1}{2}\left(\frac{\vartheta \bar{v}_{i}}{\vartheta x_{j}}+\frac{\vartheta \bar{v}_{j}}{\vartheta x_{i}}\right), \delta_{i j}$ is Kronecker's delta, and $\mu_{s g s}$ is the artificial viscosity calculated as

$$
\mu_{s g s}=-2\left(C_{s} \Delta\right)^{2}|\bar{S}|,
$$

where $|\bar{S}|=\left(2 S_{i j} S_{i j}\right)^{1 / 2}$ and $C_{s}=0.18$ are predefined values that result from assuming that the energy spectrum has an inertial range defined by the Kolmogorov $-5 / 3$ power law. An alternative model, known as the WALE model (Nicoud and Ducros, 1999), was also used to compare the LES results and to quantify the dependence on the subgrid scale modeling.

A transient PISO (Pressure Implicit Splitting of Operators) algorithm implemented in OpenFOAM (2018) was used to solve the unsteady filtered Navier-Stokes [Eq. (2)]. This algorithm, which is an improved version of the SIMPLE algorithm, contains an additional corrector step with one predictive step and two correction steps; see the work of Versteeg and Malalasekera (2007) and Oro (2012). For the time discretization, an Euler scheme was used. The spatial scheme for interpolation is Gauss linear for the gradient, Gauss linear corrected for the Laplacian, and Gauss linear upwind for the divergence. The convection term has been discretized using a second order upwind scheme.

The Volume of Fluid (VOF) method is based on the idea of a scalar fraction function $\alpha$ defined locally on each cell of the mesh whose value depends on the fraction of volume occupied by each fluid. As the two fluids A and B are separated by the free surface, the value of $\alpha$ is zero when a cell is filled with the fluid A, $\alpha=1$ when the cell is full of fluid $\mathrm{B}$, and $0<\alpha<1$ when a cell contains both fluids and the interface divides the cell. The equation to model the volume fraction of one phase $\alpha$, without mass sources or mass transfers between phases, is

$$
\frac{\partial \alpha}{\partial t}+\mathbf{v} \cdot \nabla \alpha=0
$$

Once the fraction function $\alpha$ is known, the local values of the density $\rho_{f}$ and the viscosity $\mu_{f}$ are computed for each finite volume.

\section{B. Dimensionless parameters}

The plate is submerged in the lower phase fluid of density $\rho^{b}$ and viscosity $\mu^{b}$. The upper phase has a density $\rho^{t}<\rho^{b}$ and a viscosity $\mu^{t}$. Several nondimensional numbers are used to study the problem, namely, the Reynolds number $(R e)$, Strouhal number $(S t)$, Froude number $(F r)$, density ratio $\left(\rho^{b} / \rho^{t}\right)$, and viscosity ratio $\left(\mu^{b} / \mu^{t}\right)$. The first three are defined as

$$
\operatorname{Re}_{D_{h}}=\frac{\rho^{b} \bar{U} D_{h}}{\mu^{b}}, \quad S t=\frac{f b}{\bar{U}}, \quad F r=\frac{\bar{U}}{\sqrt{g b}},
$$

where $g$ is referred as the acceleration of gravity. Its value is $9.8 \frac{\mathrm{m}}{\mathrm{s}^{2}}$. $R e_{D_{h}}$ is based on the hydraulic diameter, defined as $D_{h}=\frac{2 \cdot b \cdot c}{b+c}$ when results are compared to those presented by Satheesh and HueraHuarte (2019) or defined as $D_{h}=b$ when comparing results to those of Tian et al. (2014). In the Strouhal number, $f$ is the lift force frequency. Because the fluid properties are fixed, the nondimensional ratios $\rho^{b} / \rho^{t}$ and $\mu^{b} / \mu^{t}$ will not be changed throughout this work and are fixed to 1000 and 55.24, respectively. The hydrodynamic forces acting on the streamwise and chordwise directions, $F_{X}$ and $F_{y}$, respectively, are nondimensionalized in the form of drag $C_{D}$ and lift $C_{L}$ coefficients

$$
C_{D}=\frac{F_{x}}{\frac{1}{2} \rho \bar{U}^{2} b c}, \quad C_{L}=\frac{F_{y}}{\frac{1}{2} \rho \bar{U}^{2} b c} .
$$


TABLE II. 3D-periodic LES setup validation.

\begin{tabular}{|c|c|c|c|c|c|c|}
\hline Author & $\left\langle C_{D}\right\rangle_{t}$ & Mesh Cells & $\begin{array}{l}\text { Grids in } \\
x y \text {-plane }\end{array}$ & $\begin{array}{l}\text { Grids in } z \\
\text { direction }\end{array}$ & Scheme & $\frac{\Delta t \bar{U}}{b}$ \\
\hline Fage and Johansen (1927) & 2.13 & $\ldots$ & & $\ldots$ & Experiment & \\
\hline Tian et al. (2014) & 2.20 & 9868800 & 102760 & 96 & Smagorinsky & $6 \times 10^{-4}$ \\
\hline Present work & 1.90 & 1450880 & 45500 & 32 & Smagorinsky & $5 \times 10^{-4}$ \\
\hline Present work & 2.42 & 4863600 & 108410 & 45 & Smagorinsky & $5 \times 10^{-4}$ \\
\hline Present work & 2.34 & 5709150 & 127260 & 45 & Smagorinsky & $5 \times 10^{-4}$ \\
\hline Present work & 2.20 & 5709150 & 127260 & 45 & WALE & $5 \times 10^{-4}$ \\
\hline Present work & 2.2408 & 5709150 & 127260 & 45 & Smagorinsky & $6 \times 10^{-4}$ \\
\hline Present work & 2.1429 & 5709150 & 127260 & 45 & Smagorinsky & $8 \times 10^{-4}$ \\
\hline
\end{tabular}

Finally, the boundary conditions are mean velocity $\bar{U}$ for the inflow velocity, and pressure is set as zero normal gradient. A no slip boundary condition for the velocity and a zero normal gradient for pressure are imposed on the plate. The top and bottom boundaries and the two planes in the spanwise direction are considered as walls, and then no slip boundary condition for the velocity and a zero normal gradient for pressure are imposed. A zero-velocity gradient and zero-pressure condition are used for the outlet.

\section{RESULTS: VALIDATION OF THE NUMERICAL SETUP}

Before analyzing the dynamics of the 3D flow around the submerged flat plate in cross-flow with the two phases involved, a single phase scenario is studied for validation. The validation case involves the flow around a 3D-periodic flat plate normal to the stream current. An LES simulation is performed at high Reynolds numbers $O\left(10^{5}\right)$, as in the work of Tian et al. (2014). The drag coefficient, the characteristic flow frequencies, and the pressure on the plate surface will be compared to the previous numerical results and with experiments found in the literature (Fage and Johansen, 1927). Other features, such as the variation of the vortex dynamics produced at the wake of the plate with the proximity to the free surface, are also described.

The 3D-periodic case studied in the work of Tian et al. (2014) with a single fluid is compared with the corresponding solutions obtained here. The characteristic length used in this problem is the height of the plate $b$. The dimensions of the global computational domain are consequently $K=27.5 b, J=16 b$, and $c=4 b$, identical to Tian et al. (2014). The flow inlet boundary is located $7.5 b$ upstream from the center of the plate, and the flow outlet is located $20 b$ downstream from the center of the plate. The plate has a flat rectangular cross section with sharp corners and a thickness $t=0.02 b$. The Reynolds number based on $b$ is $R e=150000$, and the rest of the geometric values can be found in Table I. It is important to remark that in the geometry used in the work of Tian et al. (2014), the corners are smooth with different curvatures of different radii, and in our case, simulations are performed with sharp corners. As corners play a relevant role in this kind of flow, see Tian et al. (2014), some differences in the results are expected. A time step and mesh convergence process has been previously performed using several meshes and time steps in order to find global values such as the drag coefficient and the Strouhal number. For the sake of brevity, we are not including details of the whole extensive set of convergence tests performed; only the most representative results are referenced. The $3 \mathrm{D}$ computational domain has the same dimensions in the $x, y$, and $z$ directions, and the sharp corners have been maintained.

A brief selection with some of the meshes used during the convergence process is presented in Table II. The finest grid is computed twice using two different subgrid models, Smagorinsky and WALE, finding very little differences between them. The convergence trend in terms of the drag coefficient is clear. The time step used in these simulations $\Delta t=5 \times 10^{-4}$ is very similar to the one used by Tian et al. (2014). Results show good agreement with the experimental results described by Fage and Johansen (1927). The small differences between the results presented here are comparable to those found in the experiments and in previous computations even though the geometry is not identical because of the round corners. We evidence here how meshes with less than $4.8 \times 10^{6}$ cells [for $3 \mathrm{D}$-periodic case proposed by Tian et al. (2014)] lead to large errors in the drag coefficient.

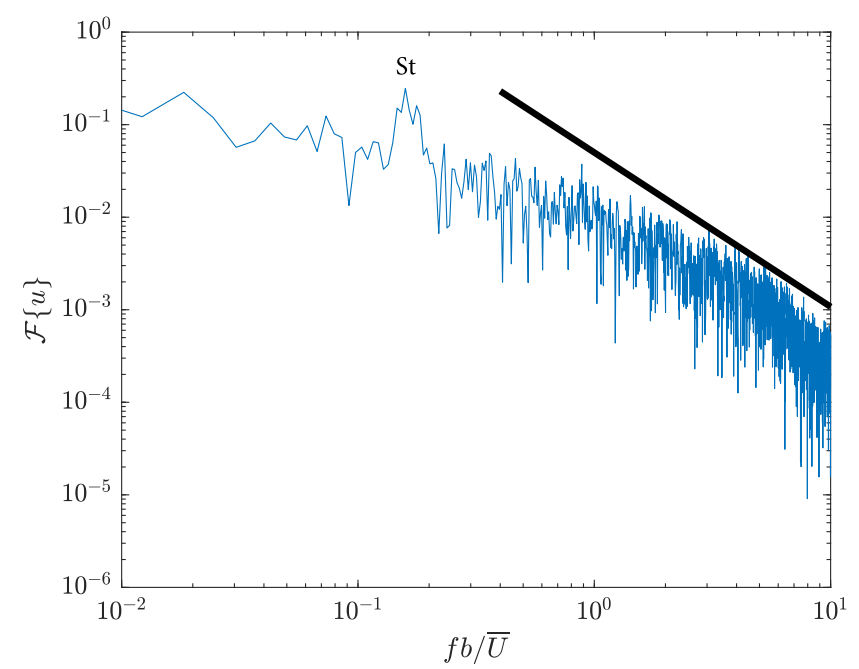

FIG. 3. Spectrum of the resolved streamwise velocity fluctuations obtained at the point $x / b=1, y / b=0.5$ and $-5 / 3$ slope. 

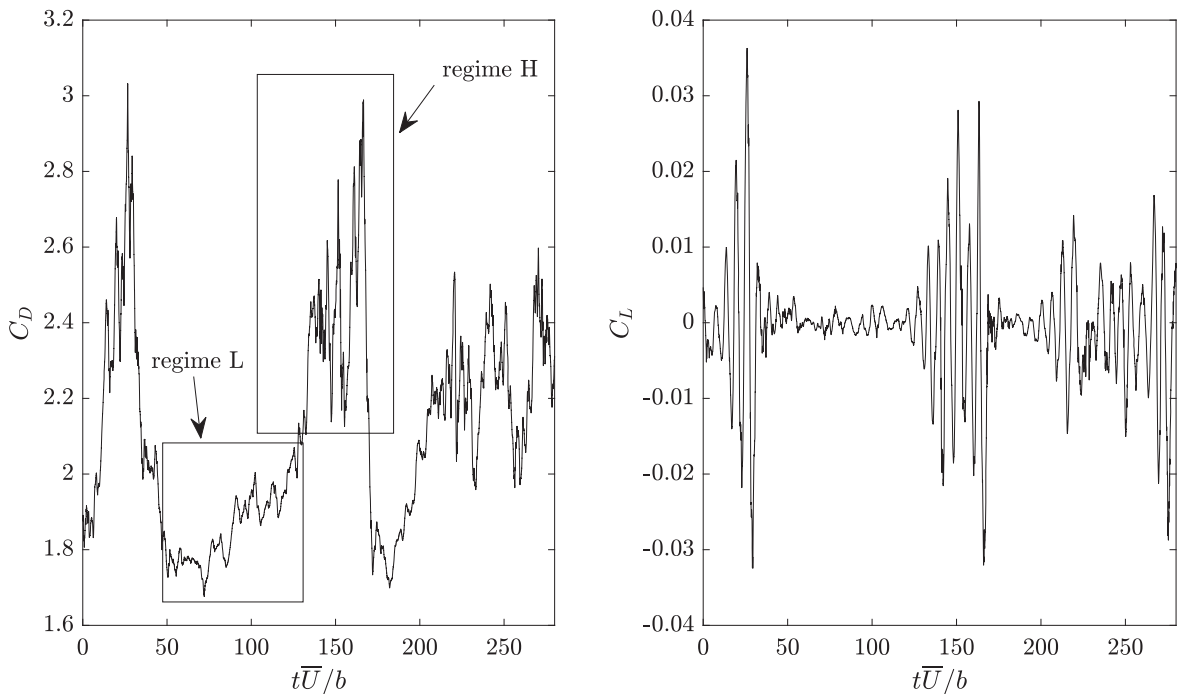

FIG. 4. Evolution of the drag (left) and lift (right) coefficients for a single fluid in the 3D-periodic case.

The spectrum of the resolved chordwise velocity fluctuations obtained at the midspan point $x / b=1, y / b=0.5$ is shown in Fig. 3. As shown, the resolved scales appear to reach the inertial subrange, with a slope of approximately $-5 / 3$ (Kravchenko and Parviz, 2000), which indicates that the turbulence spectrum has been correctly captured. In Fig. 3, a clear peak $S t=0.169$ can be identified with the primary vortex shedding frequency, which is very close to the value 0.155 computed in the work of Tian et al. (2014).

The evolution of the drag $C_{D}$ and lift $C_{L}$ coefficients is plotted in Fig. 4. Similarly to what was first presented by Najjar and Balachandar (1998), a high (H) and a low (L) drag regimes can be distinguished with the drag coefficient switching between them. The geometry of the plate corners has a very important role in the time evolution of the drag and lift coefficients, as shown by Tian et al. (2014). The authors reported how similar plates with different corners showed very different power spectra and characteristic frequencies. Despite this geometrical difference, as presented in Fig. 4, results are very similar to the ones in the work of Tian et al. (2014). Particularly, the drag and lift ranges observed, $C_{D} \in[1.7,3]$ and $C_{L} \in[-0.32,0.3]$, and their corresponding time averaged values of 2.34 and $9.97 \times 10^{-6}$ for drag and lift, respectively, compare very well with the magnitudes reported by Tian et al. (2014). Another important feature observed by Tian et al. (2014) and observed as well in the present results is that the drag and lift fluctuations are closely related, with high drag intervals associated with large fluctuations of lift coefficient, as can be seen in Fig. 4.
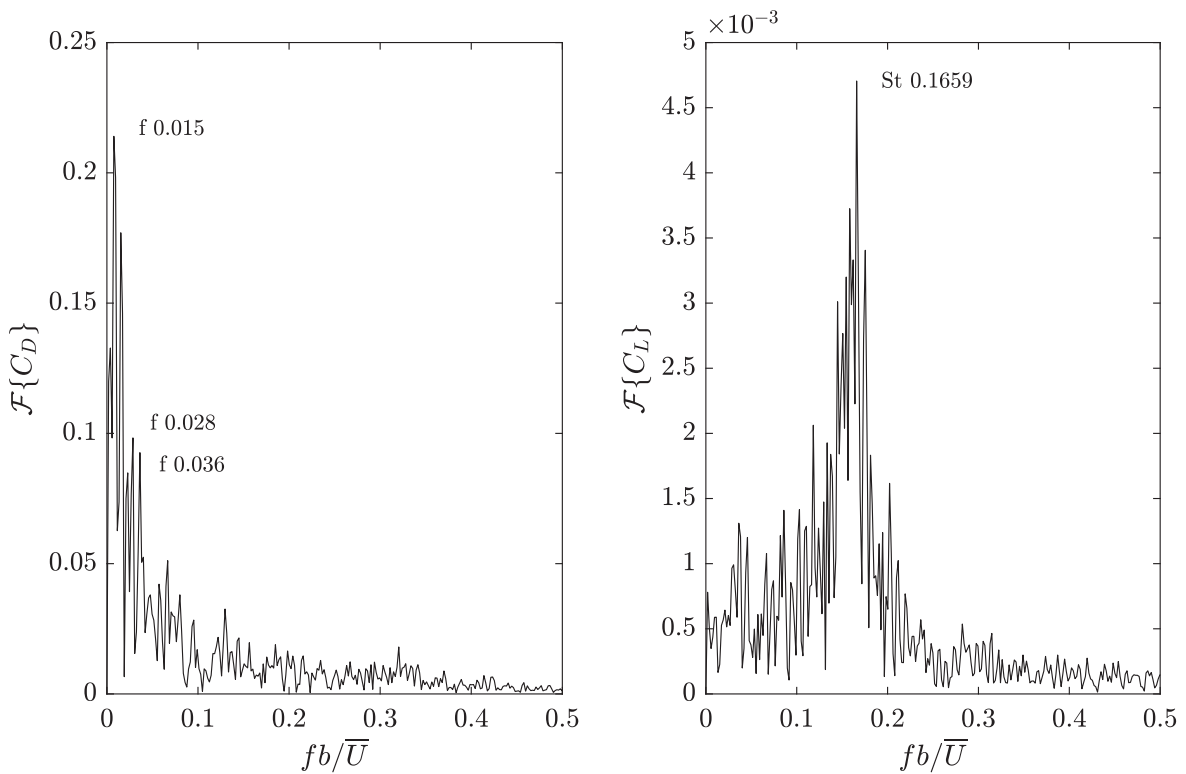

FIG. 5. Power spectra of the time evolution of the drag (left) and lift (right) coefficients for the single fluid $3 \mathrm{D}$-periodic case. 


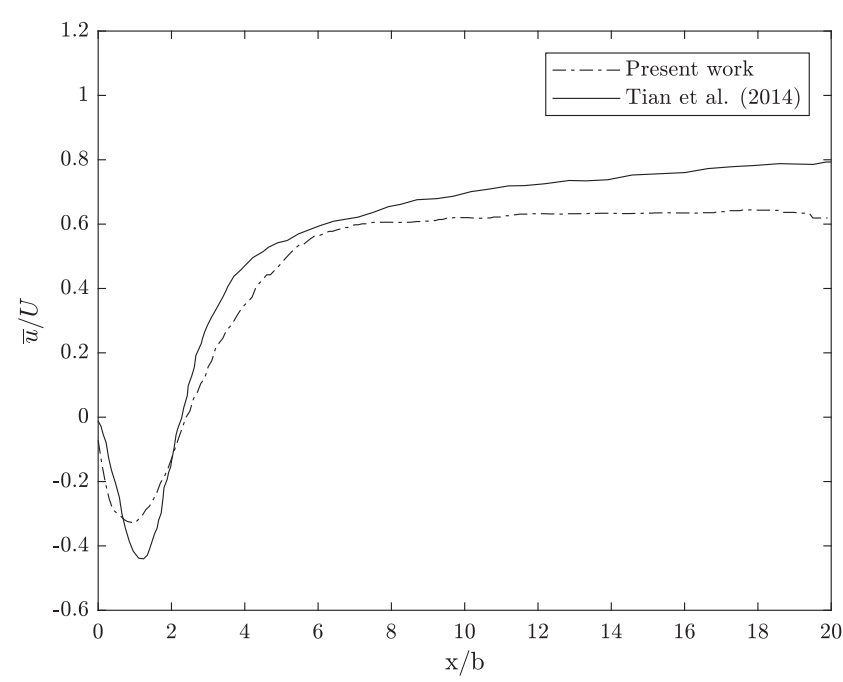

FIG. 6. Time and spanwise averaged streamwise velocity $u$ along the wake centerline $y=0$.

The power spectrum of the $\operatorname{drag} C_{D}$ and lift $C_{L}$ coefficients is plotted in Fig. 5. The low frequency unsteadiness that appears clear in the figures was also observed by Tian et al. (2014), Najjar and Balachandar (1998), and Najjar and Vanka (1995), with the values of the amplitudes and the frequencies of the drag and lift spectra, being similar to the ones obtained at the same Reynolds number by Tian et al. (2014).

The distribution of the time and spanwise averaged streamwise velocity $\langle\bar{u}\rangle / \bar{U}$ along the wake centerline $y=0$ is shown in Fig. 6. This curve shows the same trend as in the work by Tian et al. (2014), where the velocity changes its sign from negative to positive at the

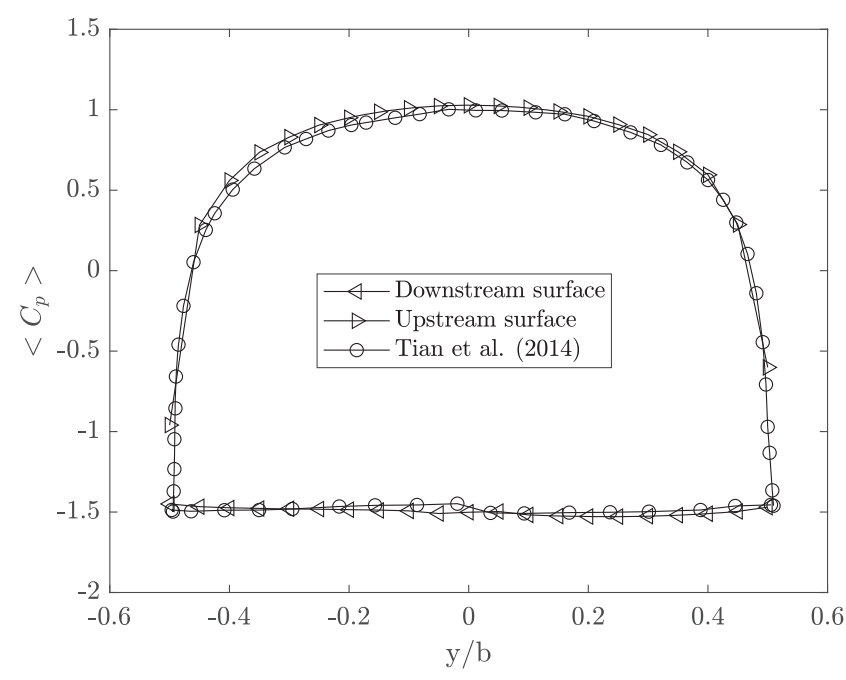

FIG. 7. Time and spanwise mean pressure coefficient $\left\langle C_{p}\right\rangle$ distribution on the front and back sides of the plate for the 3D-periodic case. end of the recirculation bubble, $x / b \approx 2.4$. For larger values of $x$, the averaged streamwise component grows and finally stabilizes its value.

Figure 7 shows the mean pressure coefficient $\left\langle C_{p}\right\rangle$ distribution, with $C_{p}=\frac{p-p_{\infty}}{\frac{1}{2} \rho U^{2}}$, on the upstream and the downstream sides of the rigid plate. The results by Tian et al. (2014) obtained with plates with smooth corners are also plotted for comparison. As it can be observed, the $\left\langle C_{p}\right\rangle$ distributions are very similar. For the present work, the pressure is just represented on both sides of the plate and not on the lateral surfaces, and as a consequence, a jump appears at $y / D= \pm 0.5$. As we explained before, the small differences found in the pressure distribution could be justified by the geometrical differences due to the presence of round corners in the work by Tian et al. (2014).

\section{RESULTS: FULL 3D SIMULATIONS INVOLVING FREE SURFACE}

In this section, 3D LES numerical simulations are conducted on the rectangular flat plate in cross-flow near the free surface. A previous validation of this code in a typical free surface problem can be found in the work of González-Gutiérrez et al. (2019). The work presented here is inspired by the experiments described in the work of Satheesh and Huera-Huarte (2019), where a rectangular flat plate was towed in a water tank with a cross section of $0.6 \times 0.6 \mathrm{~m}^{2}$. In our computational domain, dimensions match those in the experiments as indicated in Fig. 1.

The aspect ratio of the plate is defined as $A R=b / c$, where $b$ is the vertical dimension and $c$ is the horizontal dimension (parallel to the free surface). Two aspect ratios are simulated here, in-between those appearing in the experiments by Satheesh and Huera-Huarte (2019). The first is $0.5(b=0.115 \mathrm{~m}$ and $c=0.23 \mathrm{~m})$, and the second is $1(b, c=0.16 \mathrm{~m})$, chosen because they showed a very different drag coefficient trend in the experiments. All plates have the same thickness $t=5 \times 10^{-3} \mathrm{~m}$, as detailed in Fig. 1 . Three inflow velocities have been investigated, with values $0.2,0.3$, and $0.4 \mathrm{~m} / \mathrm{s}$, leading to Reynolds numbers of $30 \times 10^{3}, 45 \times 10^{3}$, and $60 \times 10^{3}$, if based on the hydraulic diameter, and corresponding Froude numbers of $0.16,0.24$, and 0.32 , respectively. Following the experimental design by Satheesh and Huera-Huarte (2019), for each $A R$, the same submergence depths for the plates have been computed. Depths $(d)$ are measured from the upper edge of the plate to the undisturbed free surface. For $A R=0.5$, the depths in dimensionless form $(d / b)$ are $0,0.0870,0.2174,0.4348,0.6087,0.7826$, 1.2609 , and 1.9130, whilst for the case with the plate of $A R=1$, the depths are $0,0.0625,0.1250,0.1875,0.3125,0.6250$, and 1.25 . Note that instead of using the chord $c$ for nondimensionalization like in the experimental work by Satheesh and Huera-Huarte (2019), here and particularly in Sec. IV, the span $b$ is used as reference length.

Different meshes have been constructed following the validation exercise presented in Sec. IV. The number of cells varies from $3.5 \times 10^{6}$ to $6 \times 10^{6}$ depending on the plate aspect ratio $A R$. In order to better understand the effect of the free surface, a case in which the free surface is replaced by a horizontal solid wall has been also included. For this case, the distance $d / b$ represents the distance from the top of the plate to the solid wall. 

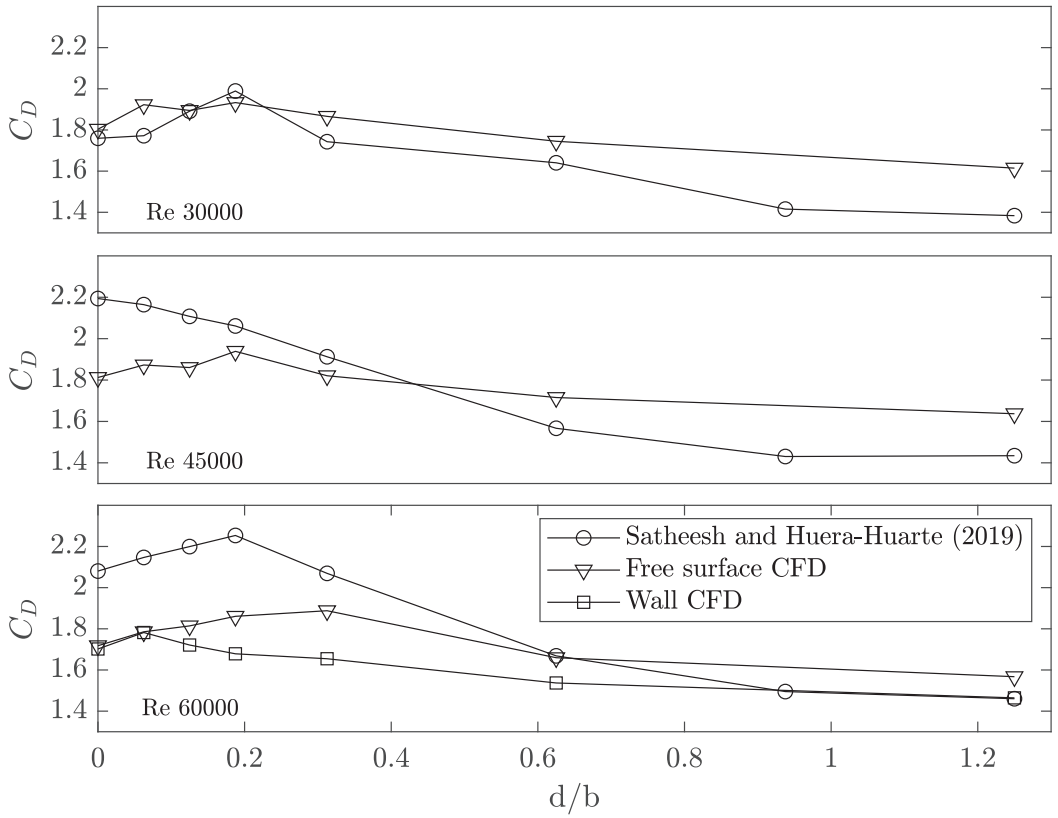

FIG. 8. Averaged drag coefficients $C_{D}$ for different depths when the plate aspect ratio $A R=1$ and $R e=30 \times 10^{3}$ (top), $R e=45 \times 10^{3}$ (middle), and $R e=60 \times 10^{3}$ (bottom). For $R e=60 \times 10^{3}$, the results for the complementary problem using a top wall instead of the free surface have been added for comparison.
The results obtained for the plate of $A R=1$ appear in Fig. 8, where a comparison between the experimental and the computational mean drag coefficient values is presented for different Reynolds numbers and submergence depths. The data suggest that the drag coefficient dependency on the Reynolds number is very small. Moreover, both the experimental and the computational results present a saturation behavior when submergence depth is large, implying a very limited influence of the free surface, as expected. In general, mean drag increases monotonically as the submergence depth is reduced from the domain center, up to a position at which drag is maximum, to start decreasing again until the upper edge of the plate is at the free surface. The numerical simulations show that the location of the maxima in drag is Re dependent but always takes place at $d / b<0.3$. The experimental data
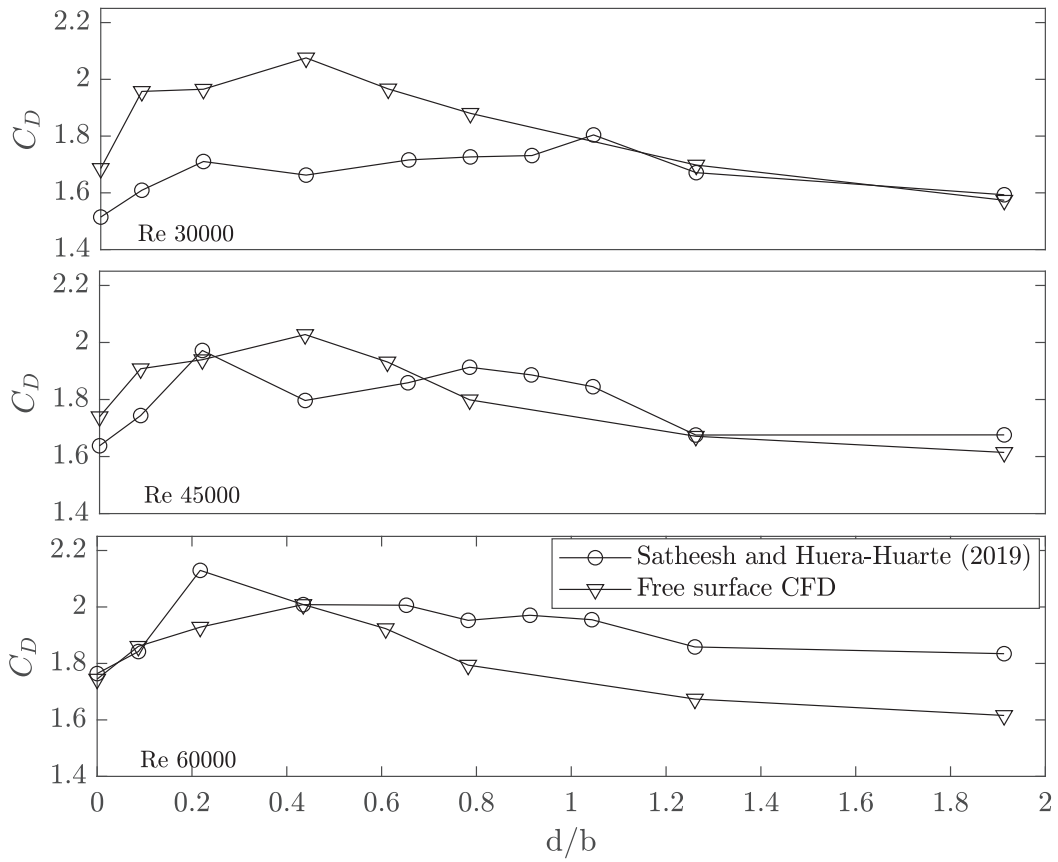

FIG. 9. Averaged drag coefficient $C_{D}$ against depth $d / b$ when the plate aspect ratio $A R=0.5$ and $R e=30 \times 10^{3}$ (top), $R e=45 \times 10^{3}$ (middle), and $R e=60 \times 10^{3}$ (bottom). 
reported in the work of Satheesh and Huera-Huarte (2019) showed how, in general, for all the aspect ratios and Re investigated, the peak in the drag coefficient took place as well at $d / b<0.3$, well in agreement with the numerical results presented here. A very similar drag trend is observed if the aspect ratio is reduced to $A R=$ 0.5; see Fig. 9. Again, as the plate is moved away of the free surface, drag increases up to a maximum to start decreasing to the stable values found at the center of the domain. In order to further understand the role of free surface, a series of numerical cases were computed after removing the problem of the gravity force and changing the free surface by a rigid wall. This was only done for the plate with $A R=1$ at $R e=60000$ as it can be seen the lower plot of Fig. 8. Under these circumstances, $d / b$ is the dimensionless distance from the top part of the plate to the fixed upper wall. The results show a similar trend in the drag coefficient if compared to the free surface case although the highest drag value takes place at a smaller submergence depth $(d / b \approx 0.0625)$. The discrepancies found in Figs. 8 and 9 could be justified by the presence of the experimental setup necessary to hold and tow the plate along the channel which has a relevant influence on the measurements, of course those elements are not considered in the numerical approach. We should also remark that no error bars are reported in the experimental results.

The analysis of the pressure distribution around the plate can help in elucidating the origin of the drag coefficient trends observed. In Fig. 10, spatiotemporal averaged dimensionless pressure coefficient $\left\langle C_{p}\right\rangle$ difference between the upstream and the downstream sides of the $A R=1$ plate along $y / b$ appears for the different depths, at $R e=60000$. The drag coefficient is dominated by the pressure force component, with the ratio between the pressure and the friction drag components in order $O\left(10^{5}\right)$ (Tian et al., 2014). The pressure difference between the front and the back sides of the plate, $\Delta\left\langle C_{p}\right\rangle$, can be associated with the pressure contribution to the drag value, and in order to visualize the influence of the depth on it, $\Delta\left\langle C_{p}\right\rangle$ appears

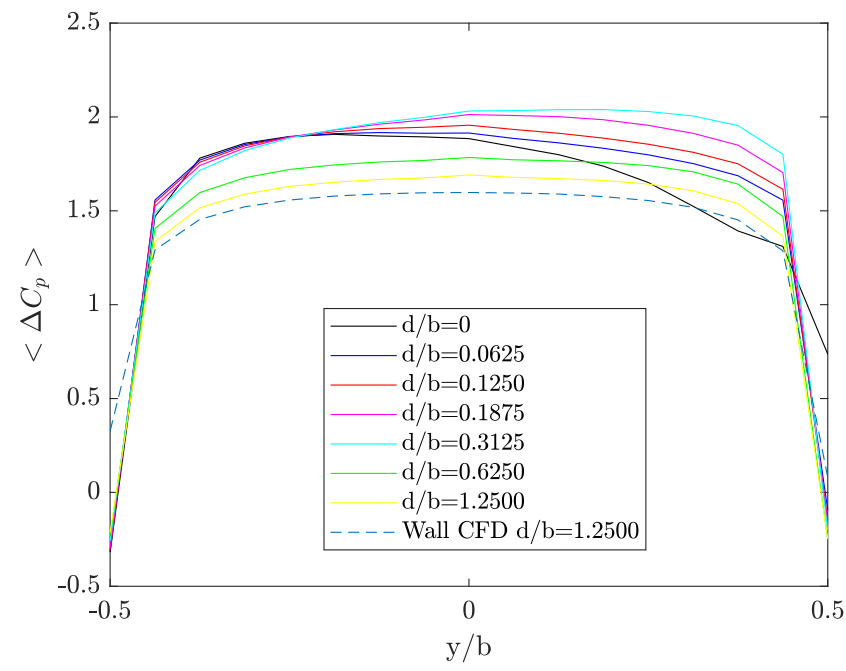

FIG. 10. Time and cross averaged dimensionless pressure coefficient $\left\langle C_{p}\right\rangle$ difference between front and back sides along $y / b$ for $A R=1$ and $R e=60000$ for different depths $d / b$. plotted against $y / b$ in Fig. 10. The larger the values of $d / b$ are, the more symmetric the $\Delta\left\langle C_{p}\right\rangle$ distribution is, meaning that the free surface influence is negligible at large depths. On the other side, for low values of $d / b$, the influence of the free surface is represented by a clear asymmetry in the curve, with a clear increment on the part of the plate closer to the free surface (positive range of $y / b$ ). Another important fact is the variation of the quantity $\int_{-b / 2}^{b / 2} \Delta\left\langle C_{p}\right\rangle d y$ for different immersion depths, which represents the pressure component of the drag coefficient. As it can be observed in Fig. 11, the maximum takes place at $d / b=0.3125$, which is in good agreement with the results previously shown in the lower plot of Fig. 8. The case of the plate near the solid wall is also shown in the plots for reference. The first difference is that due to the absence of the hydrostatic component $\left\langle C_{p}\right\rangle$, values only contain the dynamic part and the difference between the front and back sides of the plate is more evident. The values of $\Delta\left\langle C_{p}\right\rangle$ and $\int_{-b / 2}^{b / 2} \Delta\left\langle C_{p}\right\rangle d y$ are also represented. In contrast with the free surface case, the maximum value of the integral term is found at $d / b=0.0625$, with a maximum value almost equal to the case with the free surface. The trend of the curve in Fig. 12 matches well with that presented in the lower plot of Fig. 8 .

Regarding frequencies, the spectra of the resolved cross-stream velocity fluctuations obtained at the midspan for $x / b=1$ and $z / b=0$, for three different depths with dimensionless value of $0,0.125$, and 0.625, are shown in Fig. 13. According to the turbulence spectrum presented, the present simulations capture the inherent turbulent cascade where the resolved scales reach the inertial subrange described by the $-5 / 3$ Kolmogorov slope.

In Fig. 14, the evolution of the drag and lift forces is presented for the case with $A R=1$ and $R e=60000$, at the largest depth investigated of $d / b=1.25$, either with a free surface or with a solid wall. The drag values are $C_{D}=1.57 \% \pm 2.5 \%$ for the free surface case and $C_{D}=1.43 \% \pm 2.7 \%$ for the wall case. Fluctuations are very small if compared to their mean value. This result is completely different to

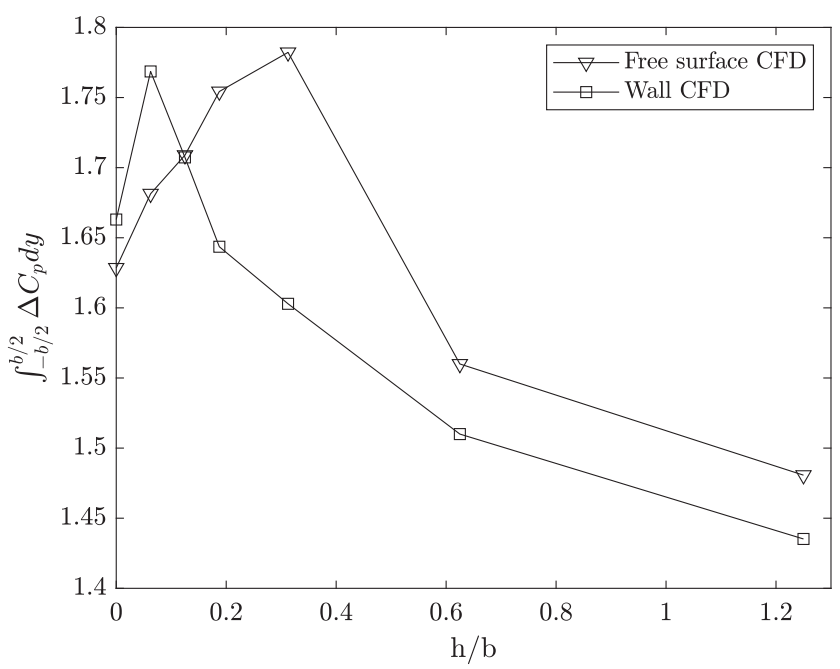

FIG. 11. Integral value of the pressure coefficient difference between both sides of the plate along the vertical direction. 

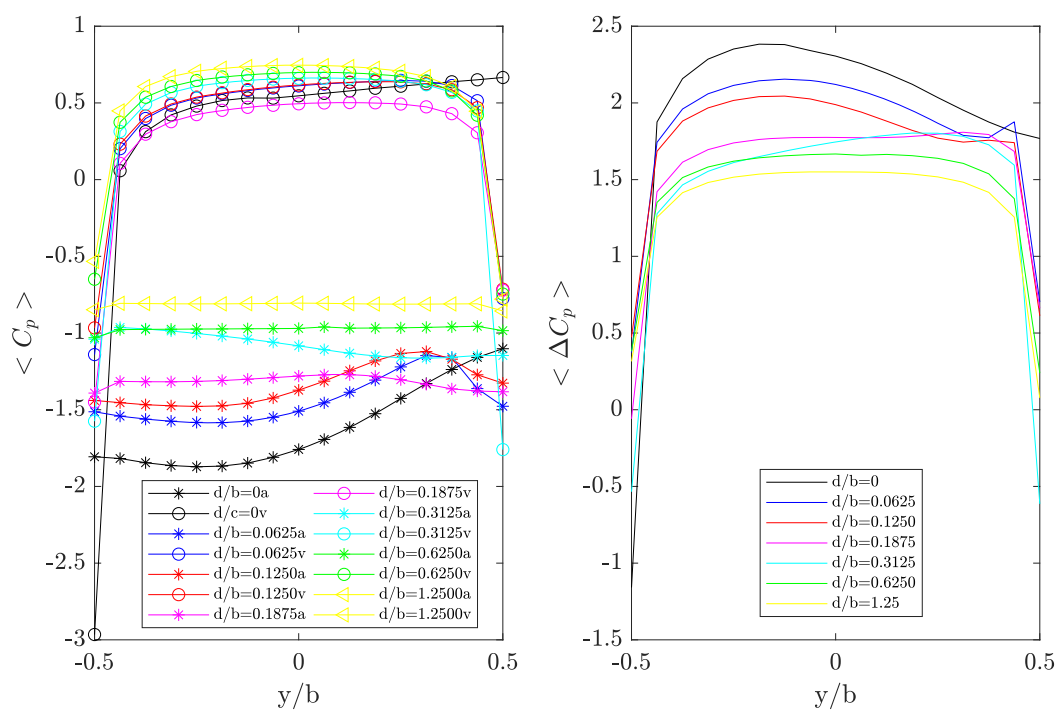

FIG. 12. Time and cross averaged dimensionless pressure coefficient $\left\langle C_{p}\right\rangle$ along $y / c$ for $A R=1$ and $R e=60000$ for different wall distances $d / b$. Circles represent the front side, $-v$ letter when the $d / b$ case is indicated, while asterisks represent the back side, a letter when the $d / b$ case is indicated. Right: Time and cross averaged dimensionless pressure coefficient $\left\langle C_{p}\right\rangle$ difference between front and back sides along $y / b$ for $A R=1$ and $R e=60000$ for different depths $d / b$.

the one shown for the periodic validation case in Sec. IV or those that appear in any of the $2 \mathrm{D}$ or $3 \mathrm{D}$-periodic cases available in the literature; see, for example, the work by Hemmati et al. (2016a) where with $R e$ of either 1200 or 2400 , two regimes $\mathrm{H}$ and L can be distinguished. In the present $3 \mathrm{D}$ simulations, with no periodic assumption between the lateral walls and conducted at higher $R e$, those regimes do not appear as the drag oscillates with a very small amplitude close to its mean value. The same applies to the case computed with the plate with a top rigid boundary, also presented in Fig. 14. In the case with the rigid wall, the drag and lift fluctuations are again very small. As expected, the amplitudes

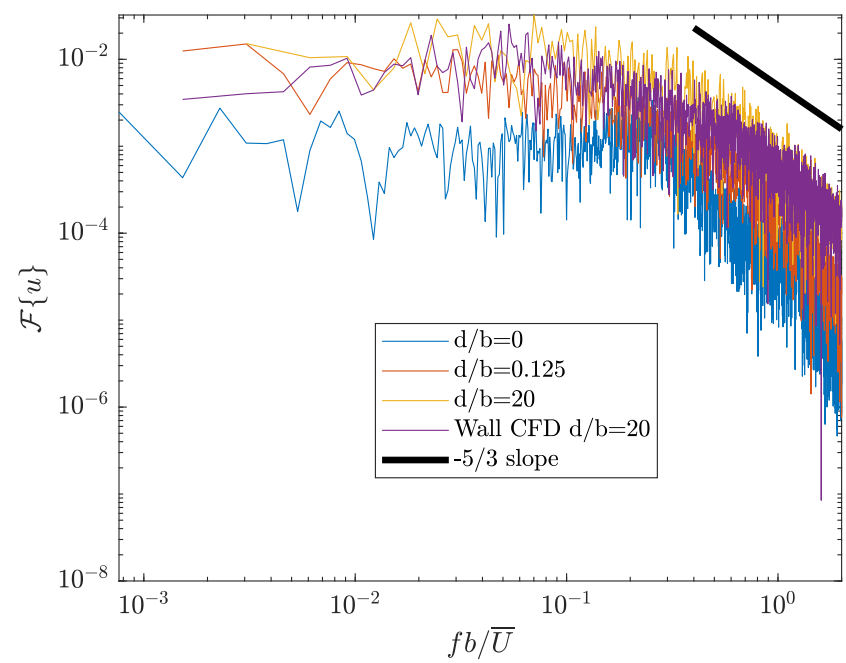

FIG. 13. Spectra of the resolved cross-stream velocity fluctuations obtained at the midspan $x / b=1$ and $z / b=0$ for three different depths; $d / b=0, d / b=0.125$, and $d / b=0.625$ when $A R=1$ and $R e=60000$. represented in the spectral analysis of the drag and lift signals, see Fig. 15, are orders of magnitude smaller than those obtained in the validation case of Sec. IV; see Fig. 5. This low amplitude spectrum does not allow to consider any characteristic frequencies to be compared to the crosswise periodic case, neither for the drag nor for the lift forces. This scenario with drag and lift forces that barely oscillate around their mean value is consistent at all depths studied.

Following the detailed analysis of the forcing terms and the comparisons made with previous experiments and numerical work, the wake dynamics is studied, with the objective to further discuss the physics of the free surface interaction with the plate. Figures 16 and 17 depict the time averaged streamline distribution for the case with the free surface and the case of the solid wall, respectively. In both figures, the Reynolds number $R e_{D_{h}}=60000$ and the aspect ratio $A R=1$. The dimensionless time window used for averaging process is $t U / b \in[0-300]$, with data that were saved every $\Delta(t U / b)=0.1$.

When the distance to the upper boundary is maximum $(d / b=$ $1.25)$, the streamlines on the wake show two symmetric counterrotating vortices with a streamwise recirculation length $L r_{x} \sim 2.5 b$. The flow at the maximum depth is practically the same for the case with the free surface (Fig. 16) and the one with the rigid wall (Fig. 17), with the main difference being the upward bending of the streamlines in the far wake for the case with the free surface, as a result of the pressure distribution created by the gravity field, which is nonexisting in the rigid wall case. Very similar results were obtained by Hemmati et al. (2016a) and Taneda and Honji (1971) for laminar flows and by Tian et al. (2014) for turbulent flows. The wake vortex dynamics change considerably when the plate approaches either the free surface or the solid wall. The counter-rotating pair tends to deform, breaking the previously observed symmetry. In the case involving the free surface, both vortices remain in the wake at all depths, but their relative positions are heavily dependent on the depth. Secondary vortices appear at the upper part of the plate, due to the free surface deformation, when depths are $0.3125,0.187$, and 

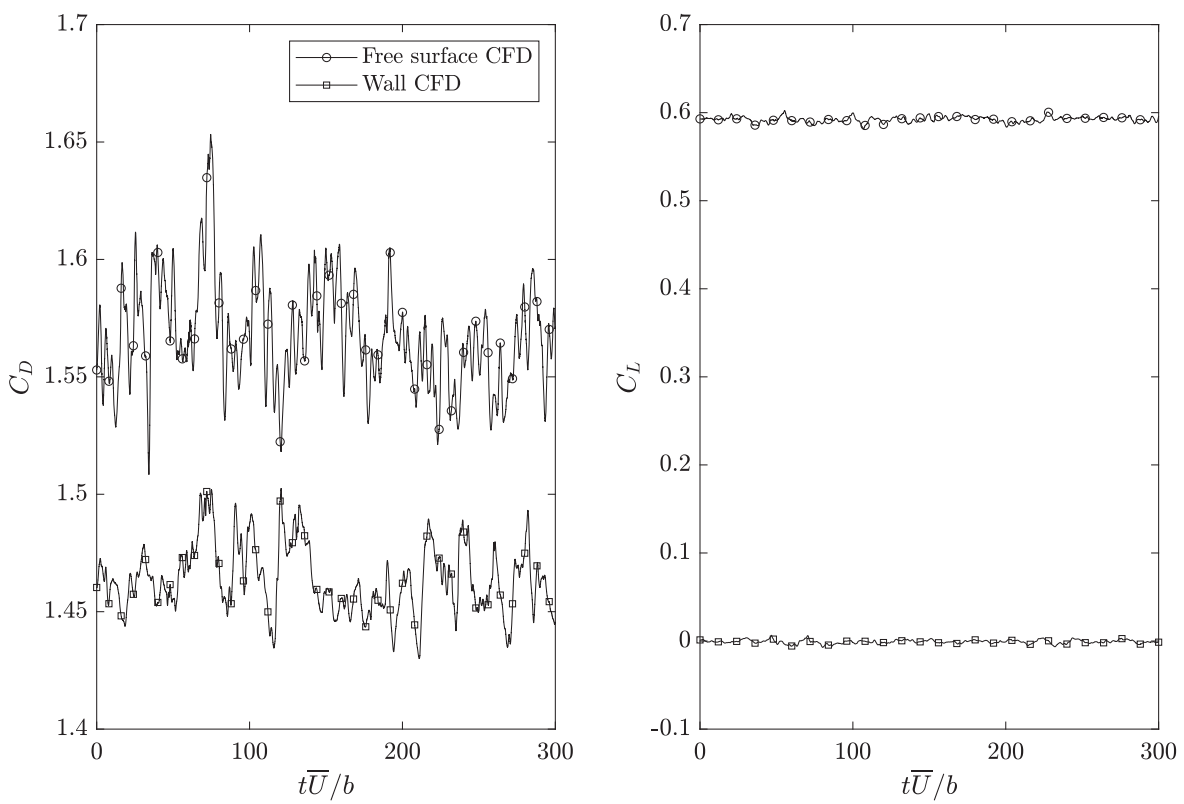

FIG. 14. Evolution of the drag (left) and lift (right) coefficients when $A R=1, R e$ $=60000$, and $d / b=1.25$.

0.1125 . At these specific depths, the main upper vortex and the secondary one form a counter-rotating pair near the free surface that creates a jetlike flow (or gap flow) accelerating the flow between the upper edge of the plate and the boundary. The free surface is able to deform and to create a small depression behind the plate that modifies the flow characteristics at the top part of the plate and allows some streamlines to overpass the plate following the free surface distortion.
With a top solid wall boundary, this complex dynamics between the counter-rotating vortices disappears at depth $d / b=0.3125$, where the upper side vortex vanishes and only the lower one dominates. If the depth is further reduced, the upper vortex appears again. Finally, when the plate contacts the wall, this top vortex is barely observed and a large counterclockwise recirculation bubble dominates the wake. As the top boundary cannot be deformed, the flow through the gap is limited and tends to the formation of a stagnation point
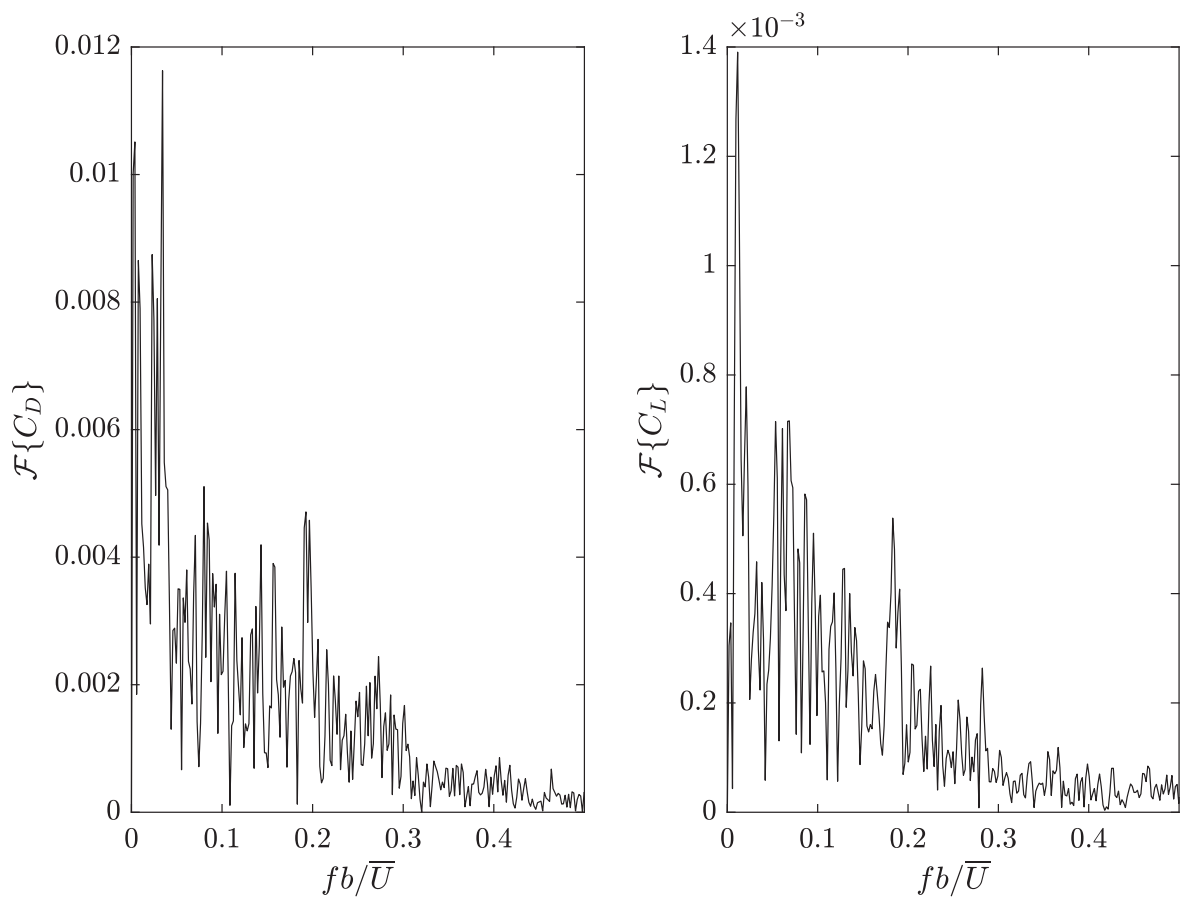

FIG. 15. Power spectra of the time evolution of the drag (left) and lift (right) coefficients when $A R=1, R e=60000$, and $d / b=1.25$. 


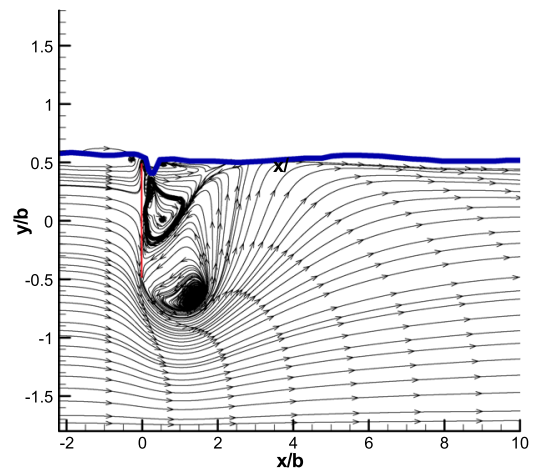

(a)

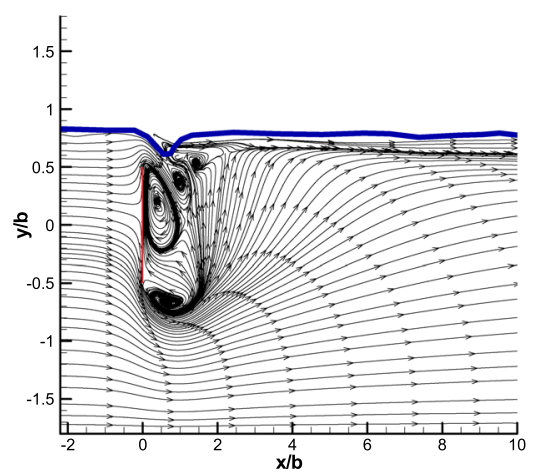

(d)

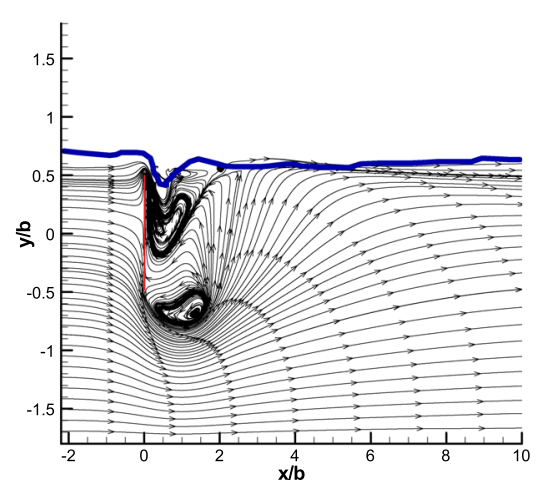

(b)

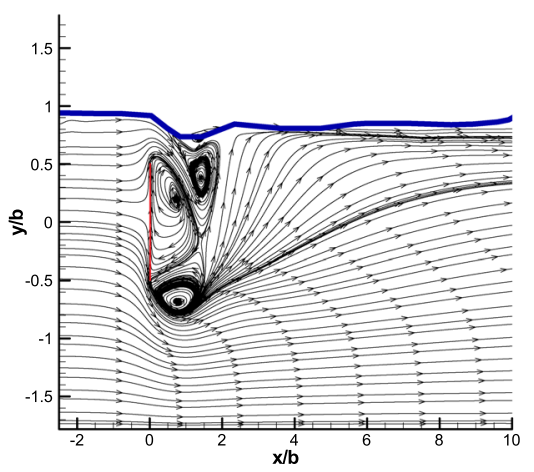

(e)

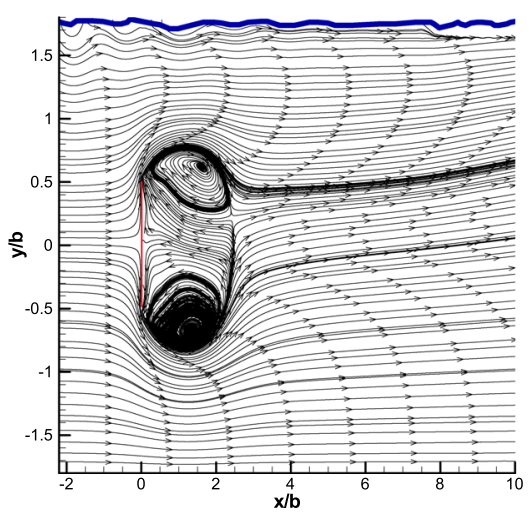

(g)

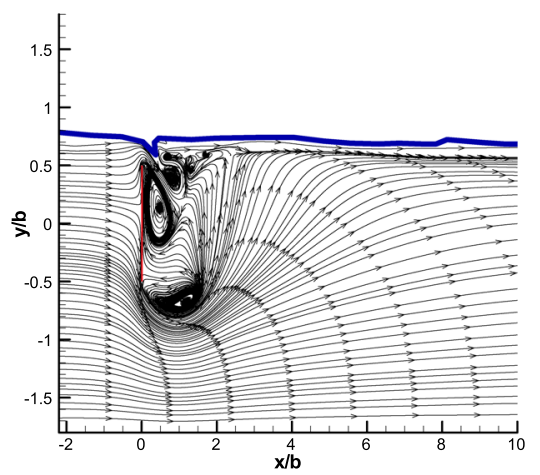

(c)

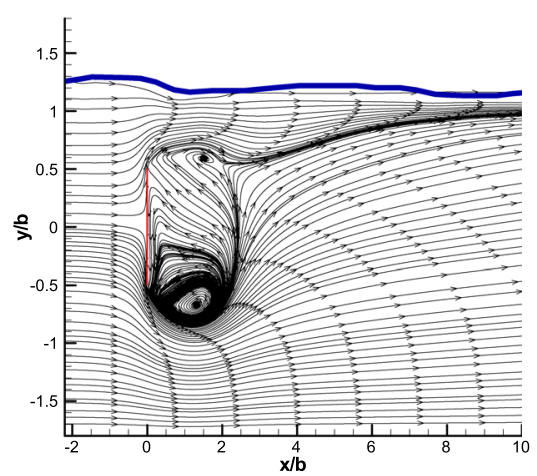

(f)

FIG. 16. Streamlines of the time and spanwise averaged flow field in the near wake at different depths $d / b$. (a) $d / b=0$, (b) $d / b=0.0625,(c) d / b=0.1125$, (d) $d / b=0.187$, (e) $d / b=0.3125$, (f) $d / b=0.625$, and $(g) d / b=1.25$.

as the gap decreases. In the limit case $d / b=0$, no flow circulates between the top part of the plate and the solid wall.

In Fig. 18, initial formation and steady state snapshots of the computed dimensionless spanwise stage of the vortex $\omega_{z}$ are compared to the experimental results shown by Satheesh and HueraHuarte (2019) for depths $d / b$ of $0,0.217,0.434$, and 1.913 for the plate of $A R=0.5$ and $R e_{D_{h}}=45000$. The dimensionless size of the PIV domain reported by Satheesh and Huera-Huarte (2019) was limited to $(y / b, z / b) \in[-1,1] \times[0,2.5]$ because of the measurement setup, whilst the computational domain shown here is larger $(x / b$, $y / b) \in[-1.8,1.8] \times[-0.5,4]$ as the same limitations do not apply to the computations. Experiments and numerical simulations show 


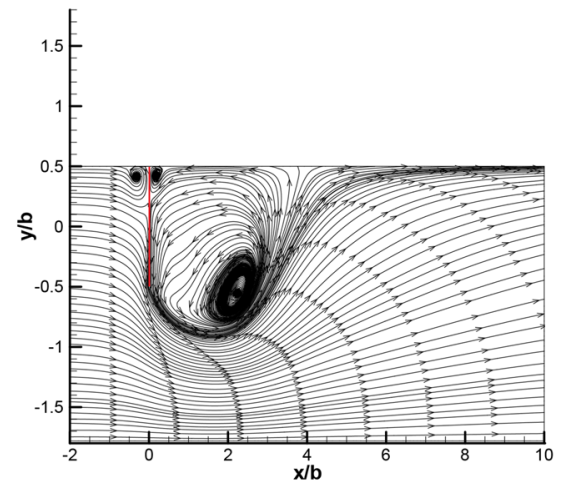

(a)

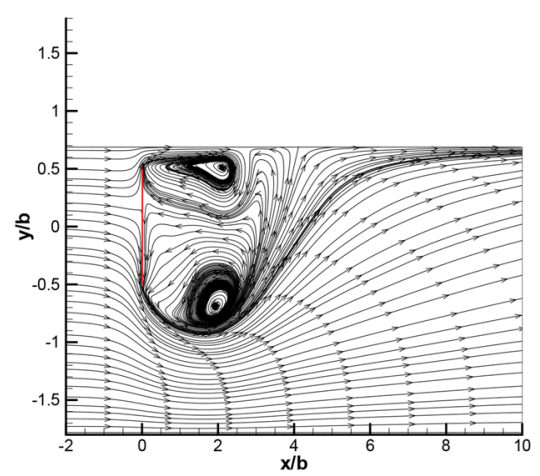

(d)

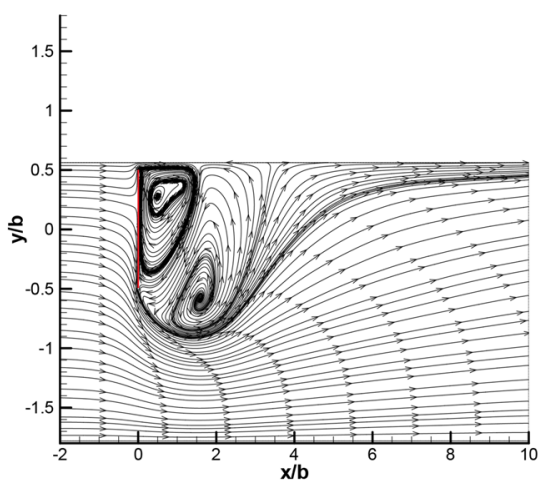

(b)

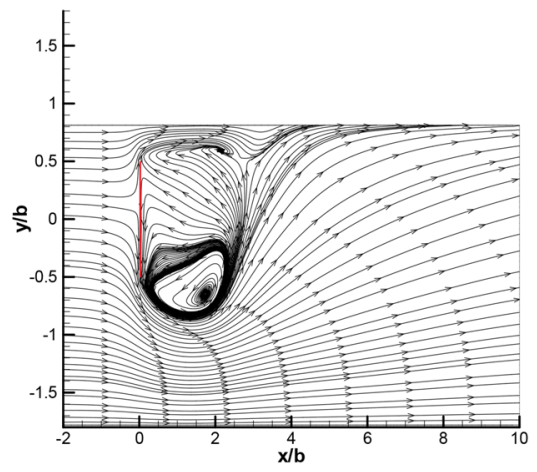

(e)

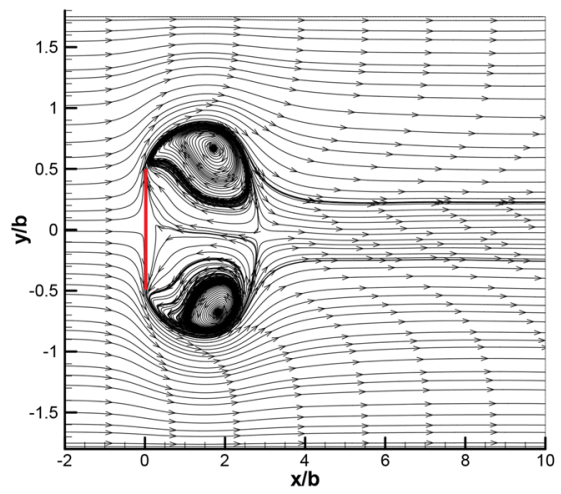

(g)

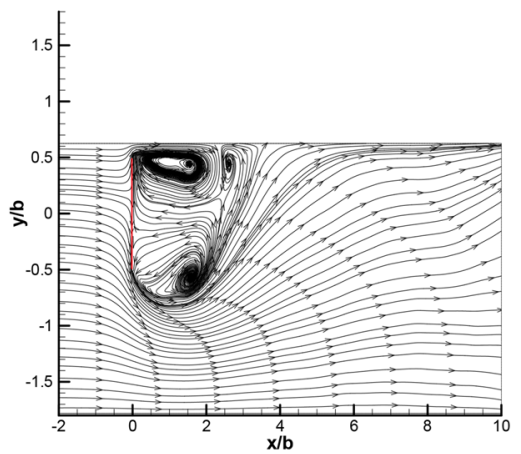

(c)

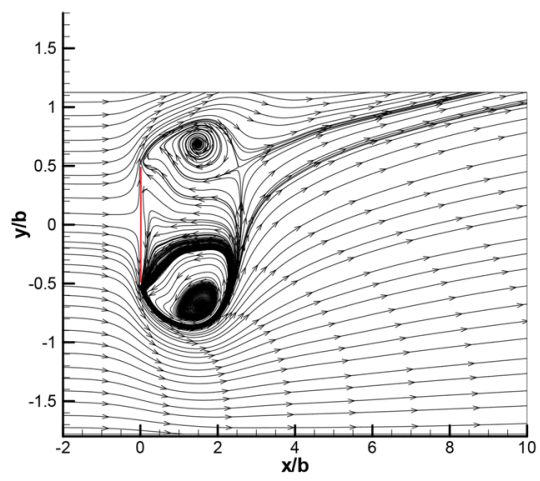

(f)

FIG. 17. Streamlines of the time and spanwise averaged flow field in the near wake at different wall distances $d / b$. (a) $d / b=0,(b) d / b=0.0625,(c) d / b=0.125,(d) d / b=$ 0.1875 , (e) $d / b=0.3125$, (f) $d / b=0.625$, and $(g) d / b=1.25$.

the same features. Vorticity tends to have a symmetric distribution as depth is increased; see $d / b=1.913$ in Fig. 18 as an example. As the plate is moved closer to the free surface, the vortex distribution looses symmetry. For the cases $d / b=0.434$ and $d / b=0.217$ in Fig. 18, the vorticity created near the free surface modifies the evolution of the upper shear layer. The generation of vorticity at the deformed free surface creates the jetlike flow described before, leaving a very weak upper shear layer, and a flow channeled toward the lower part of the plate. The same phenomena take place again at a depth $d / b$ $=0.434$. When the plate is located at the free surface, with $d / b=0$, 


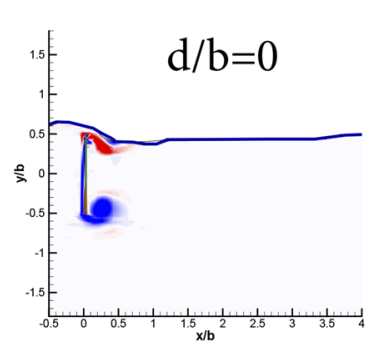

Initial state.

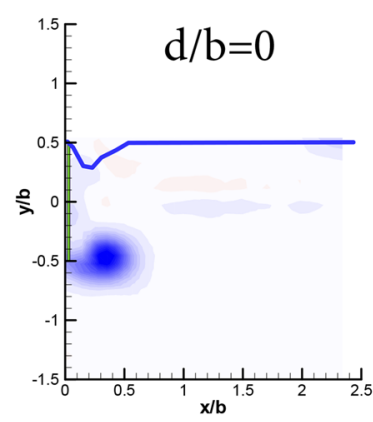

Initial state.

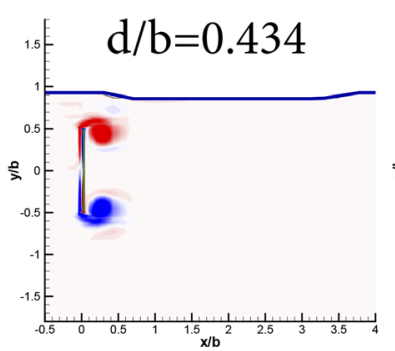

Initial state.

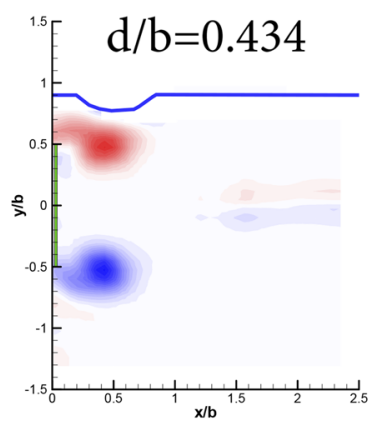

Initial state.

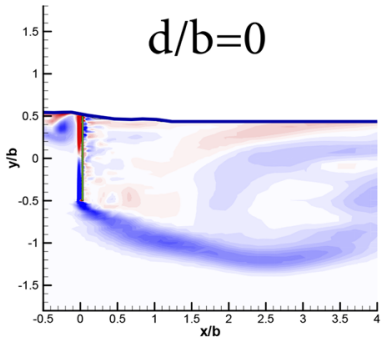

Final state.

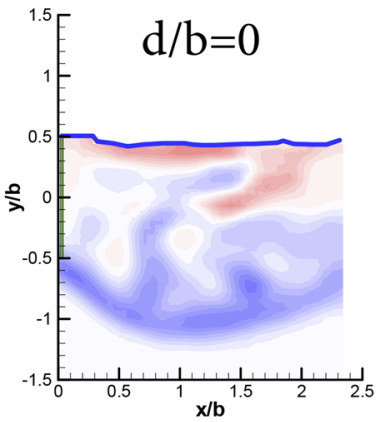

Final state.

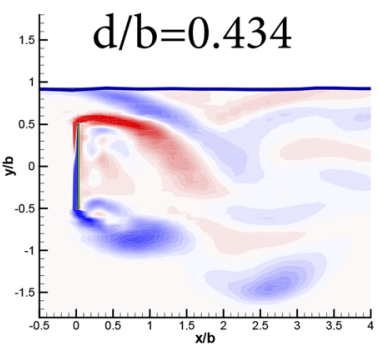

Final state.

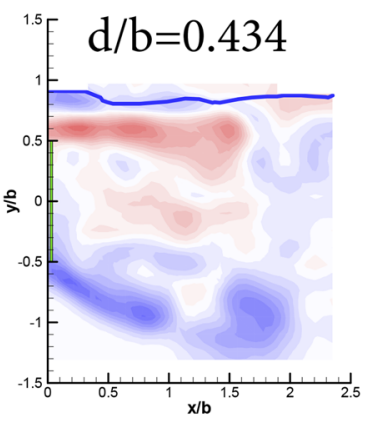

Final state.

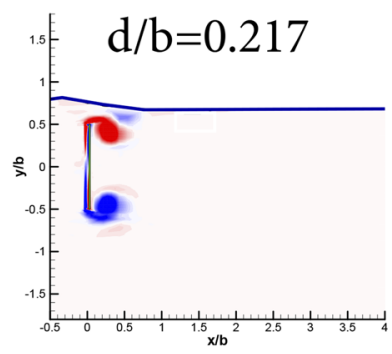

Initial state.

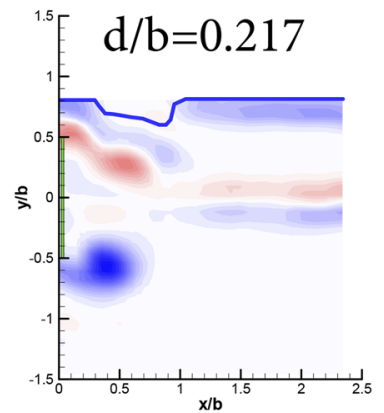

Initial state.

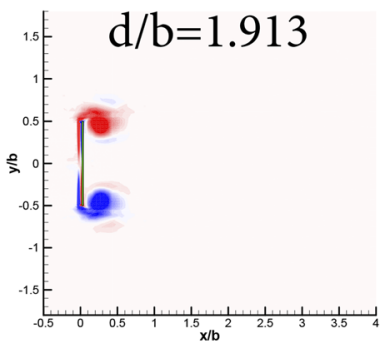

Initial state.

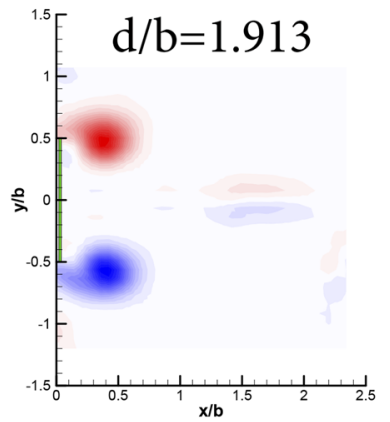

Initial state.

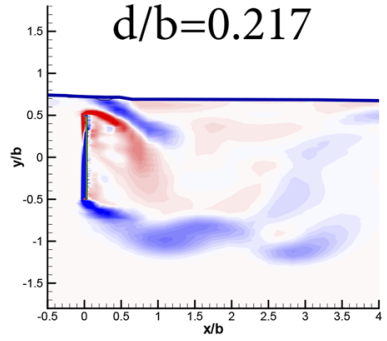

Final state.

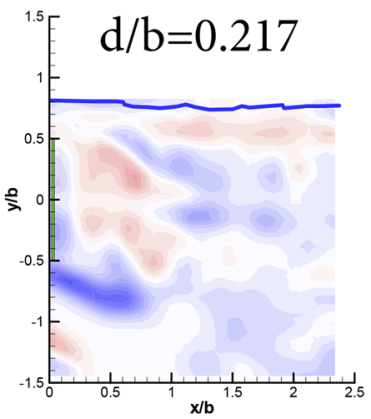

Final state.

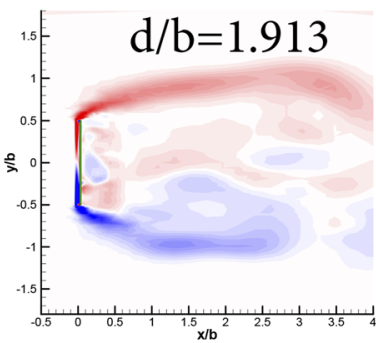

Final state.

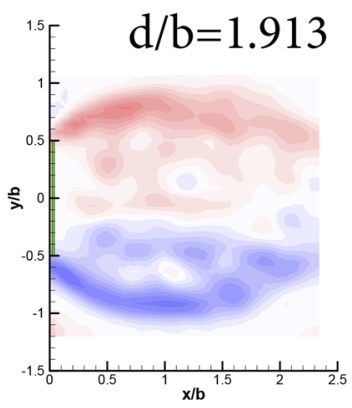

Final state.

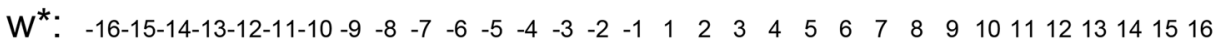

FIG. 18. Comparison between the computed dimensionless vorticity $\omega_{Z}$ (top and third rows) vs PIV experiments (second and bottom rows) in the work of Satheesh and Huera-Huarte (2019) regarding to initial and final state images for $\mathrm{d} / \mathrm{b}=0,0.217,0.434,1.913$. The color scale is $\omega_{z} \in[-16,16]$ for both results. Snapshot of the vorticity contour at when $R e=45000$ and $A R=0.5$. 
the injection of fluid through the gap flow becomes so small that no vorticity is generated in the upper part of the plate. The modification of the free surface is small as well, and the secondary vortex disappears. The size of the region that the shear layer encloses when the upper edge of plate is at the free surface is considerably bigger than in the case with $d / b=1.913$. The enlargement of this region moves away from the plate, the low pressure region, resulting in a drag reduction.

An analysis of the pressure field explains why the low (L) and high $(\mathrm{H})$ drag regimes described by Tian et al. (2014) and Hemmati et al. (2016a), at $R e=150000$ and $R e=1200$, respectively, are missing in the full $3 \mathrm{D}$ simulations presented here. In Fig. 19, an example of instantaneous dimensionless pressure field $\left(p^{*}=\frac{p}{\rho \bar{U}^{2}}\right)$ for the case of the plate with $A R=1, R e_{D_{h}}=60000$, and $d / b=1.25$ is shown. In the lower plot, instantaneous streamlines are presented for the same case. Two high and low pressure regions are found at the bow and the aft of the plate, respectively; the stability of these regions causes the low drag coefficient variation previously reported. These regions can be seen in Fig. 19, in the form of two counter-rotating vortices at $x / b \sim 1$ in the streamline plot.
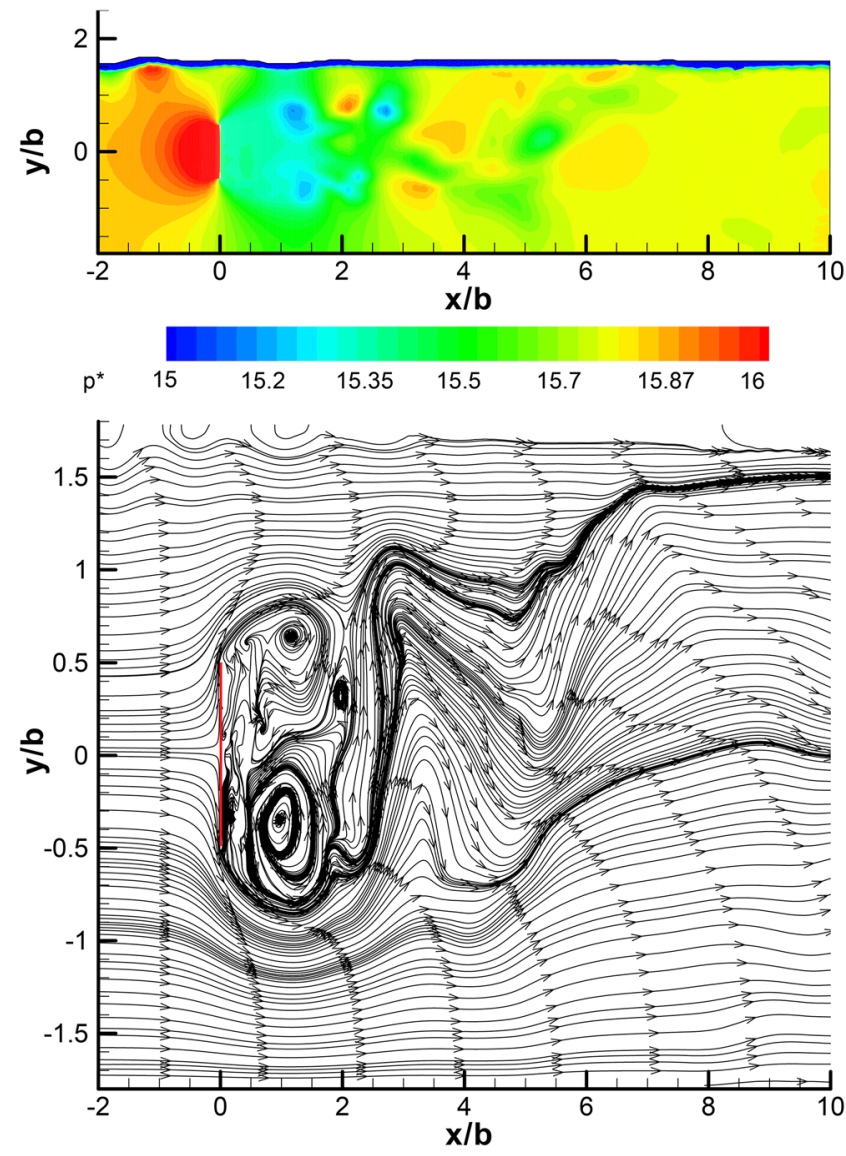

FIG. 19. Snapshot of the dimensionless pressure field $p^{*}$ (left) and streamlines at $R e=60000, A R=1$ and $d / b=1.25$.
Finally, the Q criterion (Jeong and Hussain, 1995) has been used to present the vortex structures in the turbulent wake. In particular, the differences in the flow between the case with the solid wall and its free surface counterpart are highlighted. Figures 20 and 21 show the instantaneous isosurface of vorticity $(Q=1.0)$, for cases with depth $d / b=1.25$ and $R e=60000$. Generally, the wake structures that appear in Fig. 20 are much more complex than those resulting from the 3D-periodic cases presented by Hemmati et al. (2016b) for lower Reynolds numbers in the order of 1200. A separation distance between consecutive vortex loops cannot be well identified, showing the typical complexity of a turbulent wake. The $x z$ perspective in Fig. 20 presents an alternating global structure similar to the $3 \mathrm{D}$-periodic case presented by Tian et al. (2014), where the width of the vortex street increases as the flow moves downstream; this global trend is not so evident when the free surface is considered. Vortex structures for the free surface case are stretched in the streamwise direction, whilst in the case with the rigid wall, in Fig. 20, smaller vortex structures are formed in the near wake at $x / b \sim 1$. The dynamics described by Hemmati et al. (2016b) and (2016a) appear as well here, with the alternate shedding of large scale structures at each side of the wake. The peel off process is also observed, with a streamwise flow induced away from the plate by the main vortices that carries out the smaller vortices formed on the plate edges parallel to the $z$ axis. As suggested in the work of Hemmati et al., 2016b, this process generates two secondary spanwise (out-wash) and chordwise (in-wash) flows, carrying the peeled off structures toward the wake, while pushing the newly detached structure (main vortex roller) away from the wake. Interlocked vortex-loops formed by the combination of these secondary flows are appreciated in Fig. 20. Other phenomena such as wake-split are also observed when the induced out-wash flow separates the two vortex loops formed on opposite chordwise edges of the plate, splitting the wake at its centerline. In contrast to what happens at $R e=1200$ in the work by Hemmati et al. (2016b), for larger Reynolds numbers as the ones used in this work, the peel off mechanism is not dominant and therefore no single shedding frequencies can be identified.

In Fig. 22, the Q-criteria isosurfaces are presented for the case in which the plate is in contact with the top solid boundary. The confinement effect generated by the solid wall results in a wake that is wider in both the spanwise and the chordwise directions, when compared to the case with the plate away from the wall in Fig. 20. In fact, the near wake is characterized by a large recirculation bubble, as described previously in Fig. 17 , that extends up to roughly $x / b \approx 2$, where the shear layers are opened in the wall plane from $z / b \approx-1.3$ to $z / b \approx 1.3$.

In the case with the plate being at the free surface, as in Fig. 23, an angle of $18.5^{\circ}$ is formed between the vortex structures at the free surface and the streamwise direction that can be seen in the $x z$ view of the figure. This case can be identified with any other free surface moving object, and as a consequence, this angle is close to the classical Kelvin angle $19.47^{\circ}$, always observed in the free surface footprint of navigating ships. This top view also shows how the width of the wake increases as the flow moves downstream with the Kelvin angle imposing the width and the vortex structures formed moving away from the central plane $z=0$. The wake region defined by $x / b<2$ is characterized by the formation of very small vortex 

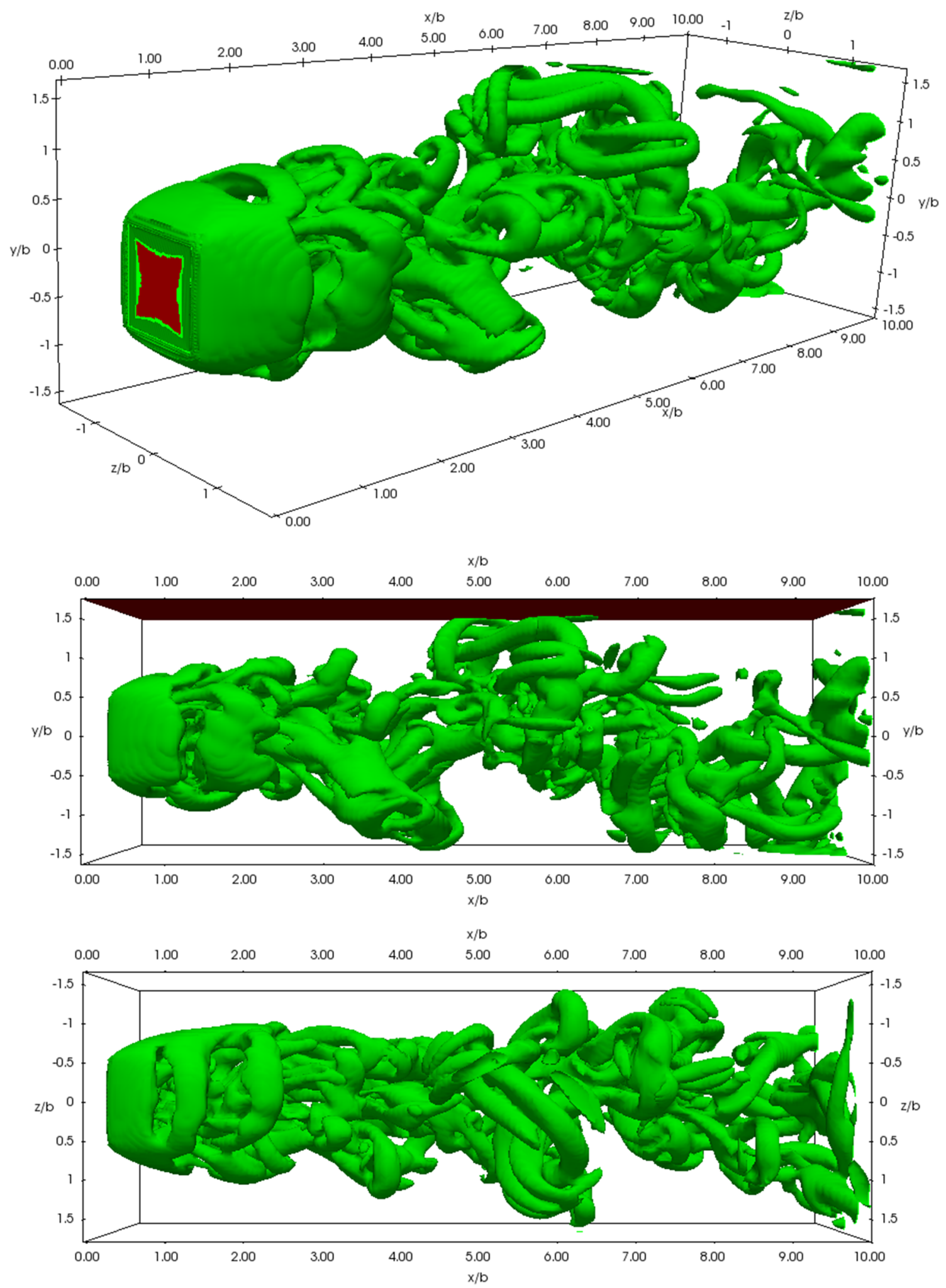

FIG. 20. Isosurface plot of $Q=1.0$ in the wake of the normal flat plate at $R e=60 k, d / b=1.25$ (deepest case) using a solid wall at the top boundary. 

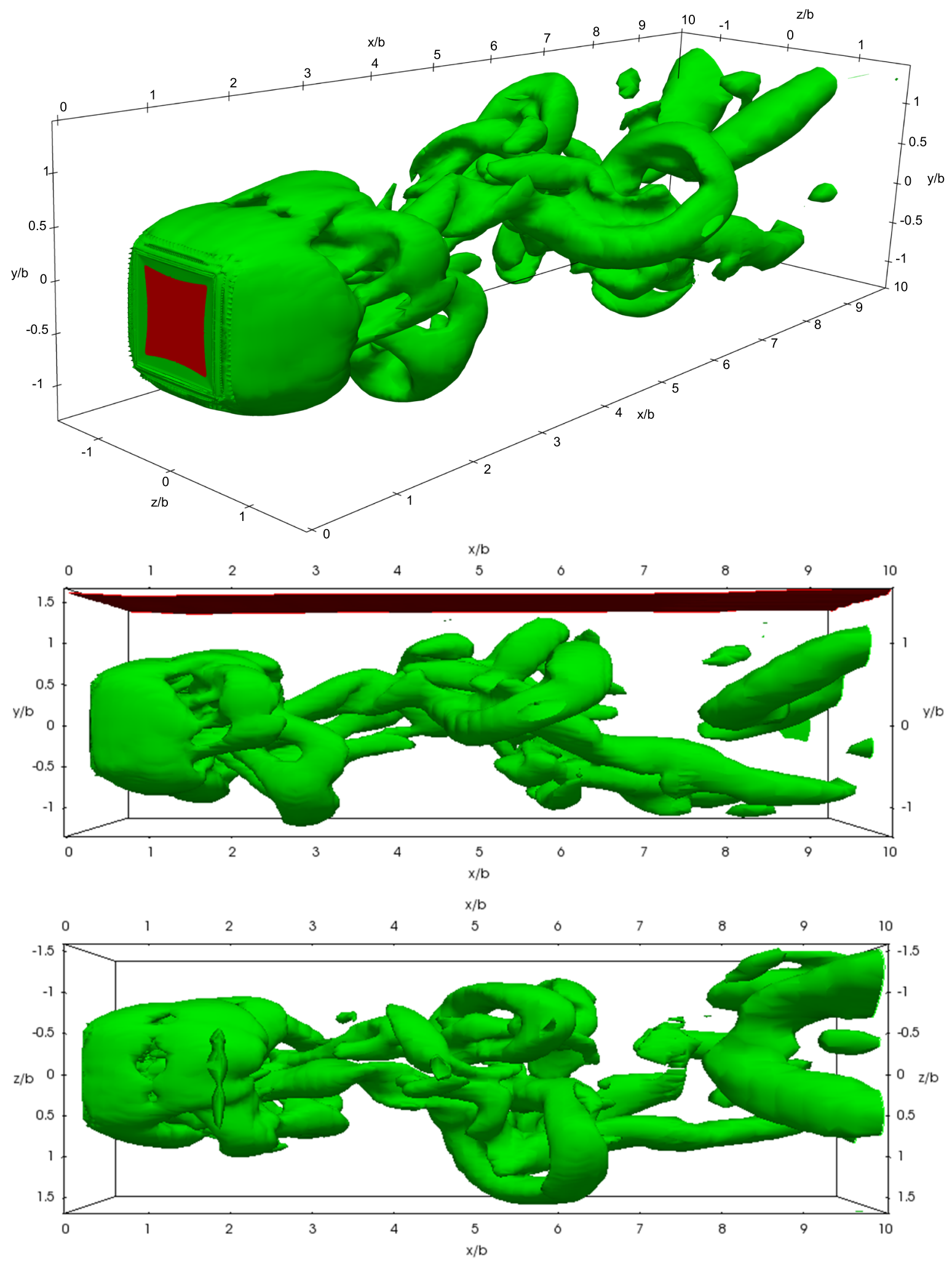

FIG. 21. Isosurface plot of $Q=1.0$ in the wake of the normal flat plate at $R e=60000, d / b=1.25$ (deepest case) with the free surface. 

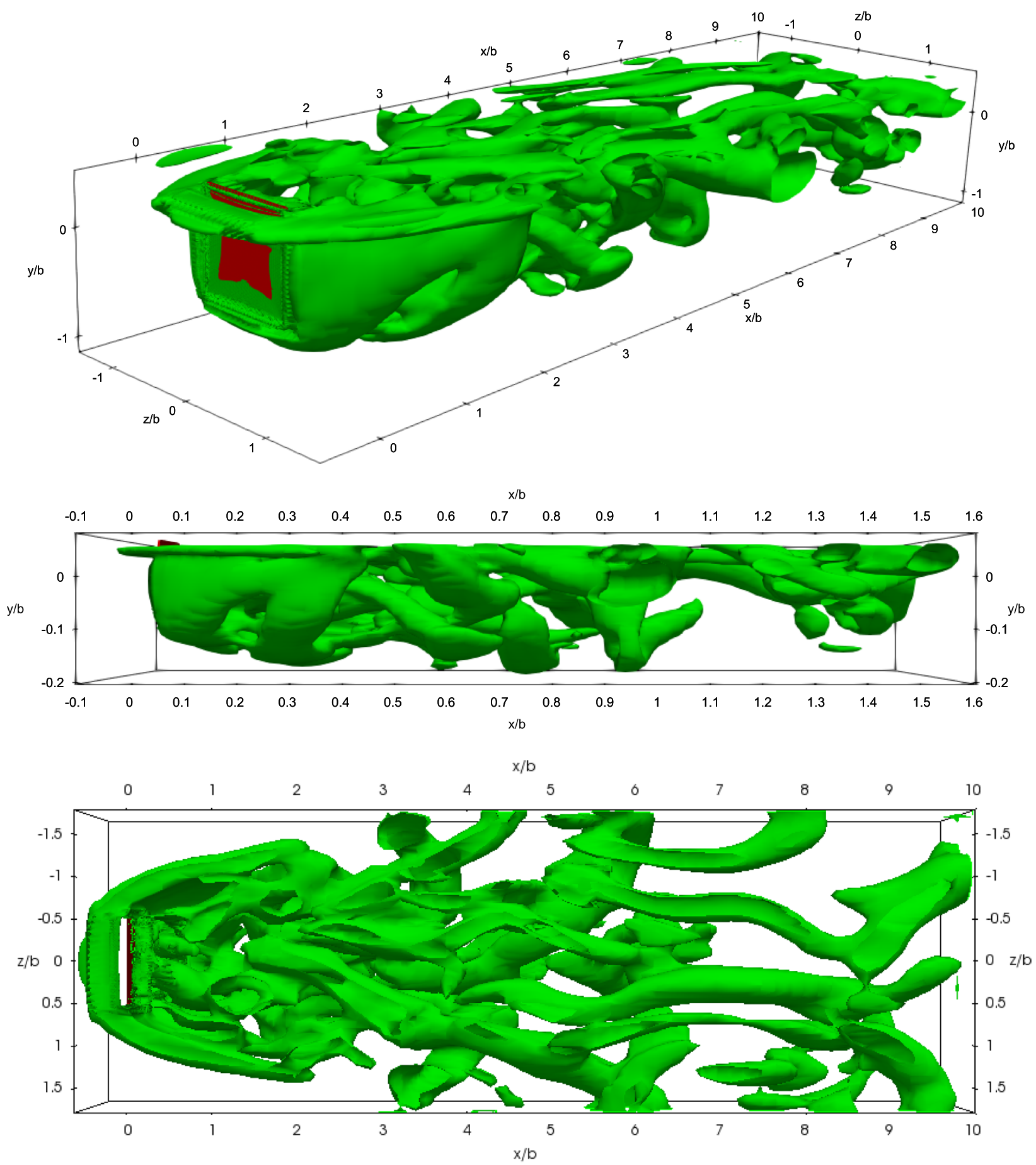

FIG. 22. Isosurface plot of $Q=1.0$ in the wake of the normal flat plate at $R e=60000, d / b=0$ using a solid wall at the top boundary.

structures at the upper edge of the plate in contact with the free surface, whilst a stable shear layer forms at the bottom part. This means that the free surface induces strong instabilities on the vortex structures coming from the top plate boundary, and as a consequence, it is difficult to identify coherent structures in the recirculation bubble.

Videos of some of the free surface flows can be downloaded from the link<https://drive.upm.es/index.php/s/J7S8PKv33x3lz9k>. 

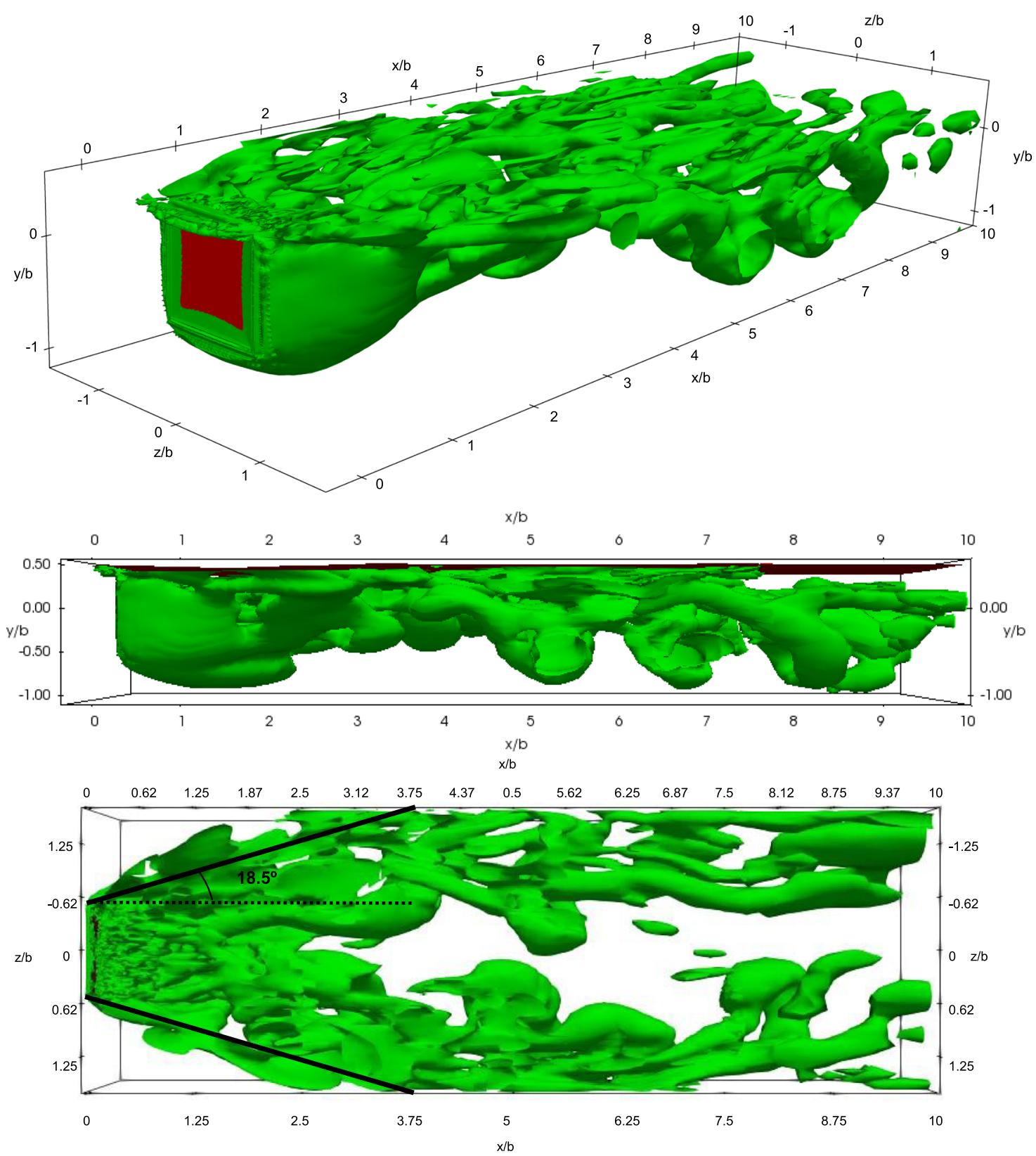

FIG. 23. Top (top) and lateral (bottom) views of the isosurface plot of $Q=1.0$ in the wake of normal flat plate at $R e=60000, d / b=0$ with the free surface.

\section{CONCLUSIONS}

A three-dimensional large-eddy simulation (3D-LES) of the flow perpendicular to a rectangular flat plate with sharp corners, in the presence of the free surface, has been conducted. The numerical study is based on nonperiodic assumptions and has been performed in the turbulent regime with $R e \sim O\left(10^{5}\right)$. The simulation includes an upper air phase and a bottom water one. The numerical problem was motivated by recent experimental work performed in a towing tank (Satheesh and Huera-Huarte, 2019). The results of the simulations showed good agreement with the experimental values available not only in terms of drag coefficients but also in terms of the main wake structures observed. A single phase case where the free surface is replaced by a rigid no-slip solid wall has also been studied to shed more light on the effect of the free surface. Both results have been compared, and the main differences have been stressed out.

The characteristic unsteady frequency associated with two high and low drag regimes, observed by previous authors using 
3D-periodic simulations at moderate Reynolds numbers $R e$ $\sim O\left(10^{3}\right)$, with plates having smooth corners, disappears in the fully $3 \mathrm{D}$ turbulent case with $\mathrm{Re} \sim O\left(10^{5}\right)$ and sharp corners considered here, with no dominant frequencies found.

A critical depth $(d / b<0.3)$ between the upper part of the plate and the free surface has been found, at which the drag force reaches a maximum value that increases approximately $25 \%$ the drag value compared to the case where the free surface has no influence. In the case of the solid wall, the same trend for the drag has been found but at larger submergence depths. This drag increment is related to the pressure field around the plate and the vortex structures on the wake. The free surface deformation at depths near the critical promotes a secondary vortex that, together with the main vortex formed at the upper edge of the plate, result in a jetlike flow between them. Under this situation, the upper shear layer bends downward, bringing closer to the plate the low pressure region, therefore increasing drag. These phenomena can have important practical implications for engineering systems near the free surface.

\section{ACKNOWLEDGMENTS}

The authors also acknowledge the financial support from the Spanish Ministry for Science, Innovation and Universities (MCIU) under grant RTI2018-096791-B-C21 Hidrodinámica de elementos de amortiguamiento del movimiento de aerogeneradores flotantes

\section{REFERENCES}

Bouscasse, B., Colagrossi, A., Marrone, S., and Souto-Iglesias, A., "SPH modelling of viscous flow past a circular cylinder interacting with a free surface," Comput. Fluids 146, 190-212 (2017).

Díaz-Ojeda, H., González, L., and Huera-Huarte, F., "Fluid structure interaction simulations involving free surface," in Proccedings of the International Conference on Ocean, Offshore, and Arctic Engineering (ASME, 2018), Paper No. OMAE2018 77207.

Díaz-Ojeda, H., González, L., and Huera-Huarte, F., "On the influence of the free surface on a stationary circular cylinder with a flexible splitter plate in laminar regime," J. Fluids Struct. 87, 102-123 (2019).

Fage, A. and Johansen, F., "On the flow of air behind an inclined flat plate of infinite span,” Proc. R. Soc. London, Ser. A 116(773), 170-197 (1927).

González-Gutiérrez, L. M., Gimenez, J. M., and Ferrer, E., "Instability onset for submerged cylinders,” Phys. Fluids 31(4), 014106 (2019).

Hemmati, A., Wood, D. H., and Martinuzzi, R. J., "Characteristics of distinct flow regimes in the wake of an infinite span normal thin flat plate," Int. J. Heat Fluid Flow 62, 423 (2016a)

Hemmati, A., Wood, D. H., and Martinuzzi, R. J., "Effect of side edge vortices and secondary induced flow on the wake of normal thin flat plates," Int. J. Heat Fluid Flow 61, 197 (2016b).
Hemmati, A., Wood, D. H., and Martinuzzi, R. J., "On simulating the flow past a normal thin flat plate,” J. Wind Eng. Ind. Aerodyn. 174, 170-187 (2018).

Jeong, J. and Hussain, F., “On the identification of a vortex," J. Fluid Mech. 285, 69-94 (1995).

Kravchenko, G. A. and Parviz, M., "Numerical studies of flow over a circular cylinder at $\operatorname{Re}_{D}=3900$," Phys. Fluids 12(2), 403-417 (2000).

Liu, I.-H., Riglin, J., Schleicher, W. C., and Oztekin, A., "Flow past a plate in the vicinity of a free surface," Ocean Eng. 111, 323-334 (2016).

Malavasi, S. and Guadagnini, A., "Interactions between a rectangular cylinder and a free-surface flow," J. Fluids Struct. 23(4), 1137-1148 (2007), Bluff Body Wakes and Vortex-Induced Vibrations (BBVIV-5).

Miyata, H., Shikazono, N., and Kanai, M., "Forces on a circular cylinder advancing steadily beneath the free-surface," Ocean. Eng. 17(1), 81-104 (1990).

Najjar, F. M. and Balachandar, S., "Low-frequency unsteadiness in the wake of a normal flat plate," J. Fluid Mech. 370, 101-147 (1998).

Najjar, F. M. and Vanka, S. P., "Simulations of the unsteady separated flow past a normal flat plate,” Int. J. Num. Met. Fluids 21, 525 (1995).

Narasimhamurthy, V. D. and Andersson, H. I., "Numerical simulation of the turbulent wake behind a normal flat plate," Int. J. Heat Fluid Flow 30(6), 1037-1043 (2009).

Nicoud, F. and Ducros, F., "Subgrid-scale stress modelling based on the square of the velocity gradient tensor," Flow, Turbul. Combust. 62(3), 183-200 (1999).

OpenFOAM, https://sourceforge.net/projects/openfoam-extend/, OpenFOAM Extend, 2018.

Oro, J. M. F., Técnicas Numéricas en Ingeniería de Fluidos. Introducción a la Dinámica de Fluidos Computacional (CFD), por el Método de Volúmenes Finitos (Reverté, 2012).

Reichl, P., Hourigan, K., and Thompson, M. C., "Flow past a cylinder close to a free surface," J. Fluid Mech. 533, 269-296 (2005).

Satheesh, S. and Huera-Huarte, F. J., "Effect of free surface on a flat plate translating normal to the flow," Ocean. Eng. 171, 458-468 (2019).

Sheridan, J., Lin, J.-C., and Rockwell, D., "Flow past a cylinder close to a free surface,” J. Fluid Mech. 330, 1-30 (1997).

Smagorinsky, J., "General circulation experiments with the primitive equations," Mon. Weather Rev. 91(3), 99-164 (1963).

Taneda, S. and Honji, H., "Unsteady flow past a flat plate normal to the direction of motion,” J. Phys. Soc. Jpn. 30, 262 (1971).

Tian, X., Ong, M. C., Yang, J., and Myrhaug, D., "Large-eddy simulation of the flow normal to a flat plate including corner effects at a high Reynolds number," J. Fluids Struct. 49, 149-169 (2014).

Tian, X., Ong, M. C., Yang, J., Myrhaug, D., and Chen, G., “Three dimensional effects of the flow normal to a flat plate at a high Reynolds number," in Proccedings of the International Conference on Ocean, Offshore, and Arctic Engineering (ASME, 2012), Paper No. OMAE2012-83730, p. 847.

Versteeg, H. K. and Malalasekera, W., "An introduction to computational fluid dynamics," in The Finite Volume Method (Pearson Education, Ltd., 2007). 